\title{
Cross-comparison of the genome sequences from human, chimpanzee, Neanderthal and a Denisovan hominin identifies novel potentially compensated mutations
}

Guojie Zhang, ${ }^{*}$ Zhang Pei,' Edward V. Ball, ${ }^{2}$ Matthew Mort, ${ }^{2}$ Hildegard Kehrer-Sawatzki ${ }^{3}$ and David N. Cooper ${ }^{2}$

'Bioinformatics Department, Beijing Genomics Institute at Shenzhen, Shenzhen 518083, China

${ }^{2}$ Institute of Medical Genetics, School of Medicine, Cardiff University, Heath Park, Cardiff CF14 4XN, UK

${ }^{3}$ Institute of Human Genetics, University of Ulm, Albert-Einstein-Allee 11, $89081 \mathrm{Ulm}$, Germany

*Correspondence to: Tel: +860755 25273794; Fax: +860755 25273114; E-mail: zhanggj@genomics.org.cn

Date received (in revised form): 22nd March 2011

\begin{abstract}
The recent publication of the draft genome sequences of the Neanderthal and a $\sim 50,000$-year-old archaic hominin from Denisova Cave in southern Siberia has ushered in a new age in molecular archaeology. We previously cross-compared the human, chimpanzee and Neanderthal genome sequences with respect to a set of disease-causing/disease-associated missense and regulatory mutations (Human Gene Mutation Database) and succeeded in identifying genetic variants which, although apparently pathogenic in humans, may represent a 'compensated' wild-type state in at least one of the other two species. Here, in an attempt to identify further 'potentially compensated mutations' (PCMs) of interest, we have compared our dataset of disease-causing/diseaseassociated mutations with their corresponding nucleotide positions in the Denisovan hominin, Neanderthal and chimpanzee genomes. Of the 15 human putatively disease-causing mutations that were found to be compensated in chimpanzee, Denisovan or Neanderthal, only a solitary F5 variant (Vall 736Met) was specific to the Denisovan. In humans, this missense mutation is associated with activated protein $C$ resistance and an increased risk of thromboembolism and recurrent miscarriage. It is unclear at this juncture whether this variant was indeed a PCM in the Denisovan or whether it could instead have been associated with disease in this ancient hominin.
\end{abstract}

Keywords: Human, chimpanzee, Neanderthal, Denisovan hominin, genome sequence, potentially compensated mutations, disease

\section{Introduction}

The recent publication of the draft sequence of the Neanderthal genome ${ }^{1}$ ushered in a new age in molecular archaeology. ${ }^{2,3}$ This achievement was followed closely by the publication of the draft genome sequence (1.9-fold coverage) of a $\sim 50,000$-year old archaic hominin from Denisova Cave in southern Siberia. ${ }^{4}$ This hominin (a
'Denisovan') is thought to have been a member of a sister group of hominins to the Neanderthals with whom they lived sympatrically during the Upper Pleistocene. ${ }^{4-7}$ Denisovans appear to be more closely related to Neanderthals than humans, having diverged from Neanderthals about 640,000 years ago and from extant Africans about 804,000 years ago. ${ }^{4}$ 
Access to DNA sequence data from ancient hominins not only promises to revolutionise our knowledge of hominin relationships, but is also potentially informative in the context of exploring the molecular basis of human genetic disease. ${ }^{8,9} \mathrm{We}$ have previously cross-compared the human, chimpanzee and Neanderthal genome sequences with a set of disease-causing/disease-associated missense and regulatory mutations in order to identify genetic variants which, although apparently pathogenic in humans, may represent a 'compensated' wild-type state in at least one of the other two species ('potentially compensated mutations' $[\mathrm{PCMs}]) .{ }^{10} \mathrm{PCM}$ correspond to variants that may have been deleterious for a certain period of evolutionary time but which persisted long enough in a given population or species to have become positively selected upon the introduction of a 'compensatory' nucleotide change. .,11-14 'com $^{\text {Such }}$ compensatory changes are thought to be localised in the same gene as the PCM. ${ }^{15}$ Not only do PCMs represent excellent candidates for recent population-specific selection (with different alleles having exhibited differential functional importance in different environments), but they may also furnish us with new insights into the genetic basis of susceptibility to common diseases. ${ }^{8,14}$ Here, in an attempt to identify further PCMs of interest, we have compared a dataset of human mutations of putative pathological significance with their corresponding nucleotide positions in the Neanderthal, Denisovan and chimpanzee genomes.

\section{Methods}

\section{Human Gene Mutation Database (HGMD) dataset}

A total of 46,060 disease-causing (DMs) or disease-associated mutations had been obtained from the HGMD ${ }^{16}$ (http://www.hgmd.org) as of 13th May 2010. These data comprised 44,348 missense mutations from within the coding regions of 2,628 genes, and 1,712 single base-pair substitutions from within the regulatory regions $\left(5^{\prime}\right.$ and $3^{\prime}$ untranslated/flanking regions) of 807 genes. Some 42,595 of the mutations were disease-causing
(41,960 missense and 635 regulatory), whereas 3,465 represented disease-associated or functional polymorphisms (2,388 missense and 1,077 regulatory) (Table 1). The latter were further ascribed to three distinct subcategories: (1) DPs, comprising variants reported to be in statistically significant $(p<0.05)$ association with a particular human disease state but lacking experimental evidence of functionality - for example, from expression studies; (2) disease-associated polymorphisms with experimental evidence of functionality (DFPs) such as, for example, altered in vitro gene expression or protein function; (3) FPs that have been shown in vitro or in vivo to affect the structure, function or expression of the gene or gene product but for which no statistically significant disease association has yet been reported (see http://www.hgmd.cf.ac. $\mathrm{uk} /$ docs/poly.html for further information).

\section{Identification of PCMs}

A total of 8,280,851 nucleotide positions at which the Denisovan genome differs from either the human (NCBI36/hg18) or chimpanzee genome were downloaded from the website of the Max Planck Institute for Evolutionary Anthropology (http://bioinf.eva. mpg.de/download/DenisovaGenome/Denisova_ Neandertal_catalog.tgz). ${ }^{1,4}$ The human and the Denisovan hominin were found to exhibit the same nucleotide at $7,283,268$ positions $(87.95$ per cent), so that the human-chimpanzee mismatches must have arisen before the divergence of modern

Table I. Missense and regulatory mutations from the HGMD used in this study, categorised by mutation type and putative role in disease aetiology

\begin{tabular}{|lrrrrr|}
$\begin{array}{l}\text { Mutation/ } \\
\text { polymorphism } \\
\text { type }\end{array}$ & \multicolumn{5}{c}{$\begin{array}{c}\text { Type and putative role in disease } \\
\text { aetiology }\end{array}$} \\
\cline { 2 - 6 } & DM & DP & DFP & FP & Total \\
\hline Coding sequence & 41,960 & 942 & 295 & I,I5I & 44,348 \\
\hline Regulatory & 635 & 340 & 391 & 346 & 1,712 \\
\hline Total & 42,595 & 1,282 & 686 & 1,497 & 46,060 \\
\hline
\end{tabular}

DM, disease-causing mutation; DP, disease-associated polymorphism lacking functional evidence; DFP, disease-associated polymorphism with functional evidence; FP, polymorphism with functional evidence but lacking a reported disease association as yet. 
humans and Denisovans (termed a 'derived' or ' $D$ ' state in the Denisovan). A total of 941,947 positions (11.38 per cent) displayed the same nucleotide in both Denisovan and chimpanzee, suggesting that the respective substitutions were human specific ('ancestral' or 'A' state in the Denisovan). The remaining 55,636 positions, which display different nucleotides in modern humans, Denisovans and chimpanzees, were termed 'undefined' ('N' state). Of the 8,280,851 Denisovan nucleotide positions investigated here, there were 5,205,736 positions at which the Neanderthal was found to differ from at least one of modern human, chimpanzee and Denisovan. From these 5,205,736 sites, we identified 197 sites for which the apparent wild-type nucleotide in

Table 2. HGMD-derived mutations identified as PCMs in the Denisovan, Neanderthal and/or chimpanzee genomes

\begin{tabular}{|c|c|c|c|c|c|c|}
\hline \multirow[b]{2}{*}{$\begin{array}{l}\text { Mutation/ } \\
\text { regulatory type }\end{array}$} & \multicolumn{6}{|c|}{ Mutation type and basis of disease aetiology } \\
\hline & PCM state & DM & DP & $\mathbf{F P}$ & DFP & Total \\
\hline \multirow[t]{9}{*}{ Coding sequence } & Human & $5 / 5$ & $38 / 43$ & II/II & $17 / 18$ & $71 / 77$ \\
\hline & Neanderthal & $0 / 0$ & $\mathrm{I} / \mathrm{I}$ & $0 / 0$ & $0 / 0$ & I/I \\
\hline & Denisovan & $\mathrm{I} / \mathrm{I}$ & $0 / 0$ & $0 / 0$ & $0 / 0$ & $1 / 1$ \\
\hline & Ancient & $0 / 0$ & $\mathrm{I} / \mathrm{I}$ & $2 / 4$ & $0 / 0$ & $3 / 5$ \\
\hline & Chimpanzee & $4 / 4$ & $7 / 8$ & $2 / 4$ & $0 / 0$ & $13 / 16$ \\
\hline & Denisovan and chimpanzee & $3 / 3$ & $4 / 5$ & $0 / 0$ & $0 / 0$ & $7 / 8$ \\
\hline & Neanderthal and chimpanzee & $2 / 2$ & $4 / 6$ & $1 / 1$ & $1 / 1$ & $8 / 10$ \\
\hline & Others & $1 / 1$ & $0 / 1$ & $0 / 0$ & $0 / 0$ & $1 / 2$ \\
\hline & Total & $16 / 16$ & $55 / 65$ & $16 / 20$ & $18 / 19$ & $105 / 120$ \\
\hline \multirow[t]{9}{*}{ Regulatory } & Human & 0 & 23 & 10 & 13 & 46 \\
\hline & Neanderthal & 0 & 0 & 2 & 1 & 3 \\
\hline & Denisovan & 0 & 0 & 0 & 3 & 3 \\
\hline & Ancient & 0 & 2 & 0 & 1 & 3 \\
\hline & Chimpanzee & 0 & 5 & 5 & 4 & 14 \\
\hline & Denisovan and chimpanzee & 0 & 4 & 1 & 1 & 6 \\
\hline & Neanderthal and chimpanzee & 0 & 1 & 1 & 0 & 2 \\
\hline & Others & 0 & 0 & 0 & 1 & 1 \\
\hline & Total & 0 & 35 & 19 & 24 & 78 \\
\hline
\end{tabular}

'Human': The Denisovan nucleotide, Neanderthal nucleotide and chimpanzee nucleotide were identical to a human DM/disease-associated mutation; 'Neanderthal': The Neanderthal nucleotide was identical to the human DM/disease-associated mutation, whereas both the chimpanzee nucleotide and the Denisovan nucleotide were identical to the human wild-type nucleotide; 'Denisovan': The Denisovan nucleotide was identical to the human DM/disease-associated mutation, whereas both the chimpanzee nucleotide and the Neanderthal nucleotide were identical to the human wild-type nucleotide; 'Ancient': Both the Denisovan nucleotide and the Neanderthal nucleotide were identical to the human DM/disease-associated P mutation, whereas the chimpanzee nucleotide was identical to the human wild-type nucleotide. 'Chimpanzee': The chimpanzee nucleotide was identical to the human DM/disease-associated mutation, whereas both the Neanderthal nucleotide and the Denisovan nucleotide were identical to the modern human wild-type nucleotide. 'Denisovan and chimpanzee': Both the Denisovan nucleotide and the chimpanzee nucleotide were identical to the human DM/disease-associated mutation, whereas the Neanderthal nucleotide was identical to the human wild-type nucleotide; 'Neanderthal and chimpanzee': Both the Neanderthal nucleotide and the chimpanzee nucleotide were identical to the human DM/disease-associated mutation, whereas the Denisovan nucleotide was identical to the human wild-type nucleotide. Under coding sequence, ' $a / b$ ' means that there were a total number of ' $b$ ' mutations, of which ' $a$ ' were non-synonymous mutations (there were some synonymous mutations within the coding sequence; eg CM068I90, CM077900).

PCM, potentially compensated mutations; DM, disease-causing mutation; DP, disease-associated polymorphism with functional evidence; FP, polymorphism with functional evidence but lacking a reported disease association as yet; DFP, disease-associated polymorphism with functional evidence. 
Denisovan, Neanderthal or chimpanzee was logged in the HGMD as disease causing or disease associated in modern humans (Table 2). From the remaining 3,075,115 sites, we identified 117 sites for which the apparent wild-type nucleotide in the Denisovan or chimpanzee was logged in the HGMD as disease causing or disease associated in either the Denisovan or chimpanzee (Table 3).

\section{Gene ontology (GO) enrichment analysis}

A GO enrichment analysis of PCM-containing genes against a background of 2,688 human disease-causing genes was performed using the DAVID bioinformatics tool. ${ }^{17}$ The statistical significance of a particular GO term was calculated using Fisher's exact test, which was then adjusted to allow for multiple testing by means of the Benjamini-Hochberg correction. ${ }^{18}$

Table 3. HGMD-derived mutations identified as PCMs in the Denisovan genome and/or chimpanzee genome

\begin{tabular}{llccccc}
\multicolumn{5}{c}{$\begin{array}{c}\text { Mutation type and basis of disease } \\
\text { aetiology }\end{array}$} \\
$\begin{array}{l}\text { Mutation/ } \\
\text { regulatory } \\
\text { type }\end{array}$ & PCM state & DM & DP & FP & DFP & Total \\
$\begin{array}{l}\text { Coding } \\
\text { sequence }\end{array}$ & Ancestral & $5 / 5$ & $24 / 29$ & $9 / 9$ & $5 / 7$ & $43 / 50$ \\
& Derived & $4 / 4$ & $7 / 7$ & $4 / 5$ & $4 / 4$ & $19 / 20$ \\
\hline & Denisovan & $0 / 0$ & $4 / 6$ & $2 / 2$ & $0 / 0$ & $6 / 8$ \\
\hline & Others & $0 / 0$ & $1 / 1$ & $0 / 0$ & $0 / 0$ & $1 / 1$ \\
\hline & Total & $9 / 9$ & $36 / 43$ & $15 / 16$ & $9 / 11$ & $69 / 79$ \\
\hline Regulatory & Ancestral & 2 & 6 & 9 & 12 & 29 \\
\hline & Derived & 1 & 2 & 1 & 2 & 6 \\
\hline & Denisovan & 0 & 1 & 0 & 2 & 3 \\
\hline & Total & 3 & 9 & 10 & 16 & 38 \\
\hline
\end{tabular}

Ancestral: Both the Denisovan nucleotide and the chimpanzee nucleotide were identical to the human DM/disease-associated mutation; Derived: The chimpanzee nucleotide was identical to the human DM/disease-associated mutation, whereas the Denisovan nucleotide was identical to the human wild-type nucleotide; Denisovan: The Denisovan nucleotide was identical to the human DM/disease-associated mutation, whereas the chimpanzee nucleotide was identical to the human wild-type nucleotide. Under coding sequence, ' $a / b$ ' means there were a total number of ' $b$ ' mutations, of which 'a' were non- synonymous mutations.

PCM, potentially compensated mutations; DM, disease-causing mutation; DP, diseaseassociated polymorphism with functional evidence; FP, polymorphism with functional evidence but lacking a reported disease association as yet; DFP, disease-associated polymorphism with functional evidence.

\section{Calculation of Wright's fixation index $\left(\mathrm{F}_{\mathrm{ST}}\right)$ values}

The $\mathrm{F}_{\mathrm{ST}}$ measures the proportion of genetic diversity in a subdivided population that is attributable to allele frequency differences between subpopulations. Pairwise $\mathrm{F}_{\mathrm{ST}}$ values have also been used as a measure of genetic distance between populations. In this context, the allele frequencies of polymorphic ancestral PCMs in selected populations were obtained from HapMap (http://hapmap.ncbi. nlm.nih.gov/) and pairwise $\mathrm{F}_{\mathrm{ST}}$ values were estimated for each polymorphism using the small sample estimate proposed by Weir and Hill. ${ }^{19}$ The significance of individual $F_{S T}$ values was then assessed by reference to the empirical distribution of $\mathrm{F}_{\mathrm{ST}}$ among all single nucleotide polymorphisms (SNPs) in HapMap.

\section{Results and discussion}

\section{Identification of PCMs in the Denisovan, Neanderthal and/or chimpanzee genomes}

A total of 44,348 missense mutations from 2,628 genes and 1,712 putative regulatory mutations from 807 genes, which have been recorded in the HGMD as being either causative of (or associated with) a human inherited disease state, were crosscompared with the corresponding nucleotide positions in the Neanderthal, Denisovan and chimpanzee genomes.

When the 197 PCMs covered by both the Denisovan and the Neanderthal sequences were considered, these included 129 of 143 PCMs identified in the Neanderthal genome (10/12 DMs, 65/ 73 DPs, 25/26 FPs, 29/32 DFPs), and 123 (62 per cent) PCMs for which the Denisovan, Neanderthal and chimpanzee wild-type nucleotides were identical to the human disease-causing/disease-associated mutant allele. Of the 117 PCMs covered only by the Denisovan sequence, there were 79 (67.5 per cent) for which both the Denisovan nucleotide and the chimpanzee nucleotide were identical to a human DM/disease-associated mutation. This may be indicative of either a bottleneck effect or selection during the evolution of the modern human lineage. Of the $197 \mathrm{PCMs}$, there was one mutation 


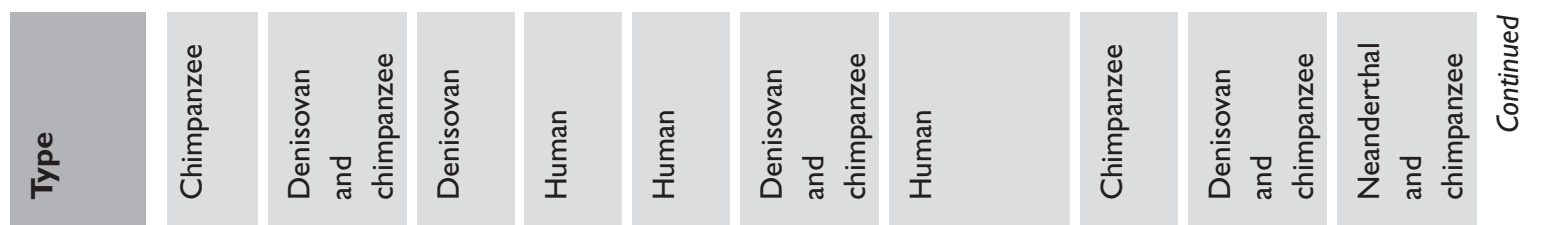

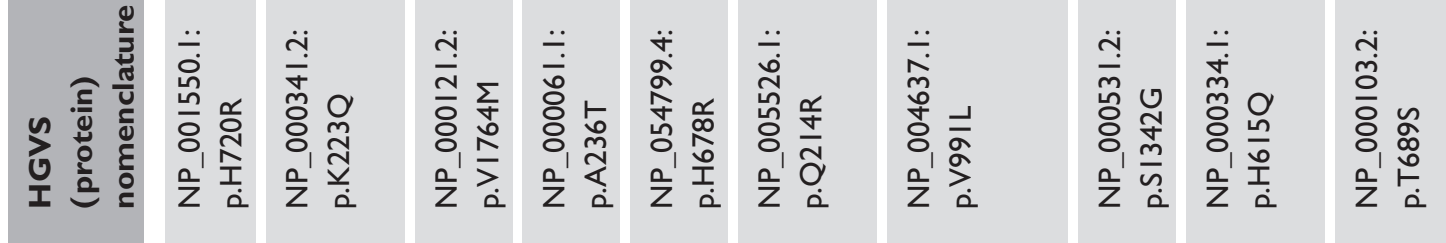

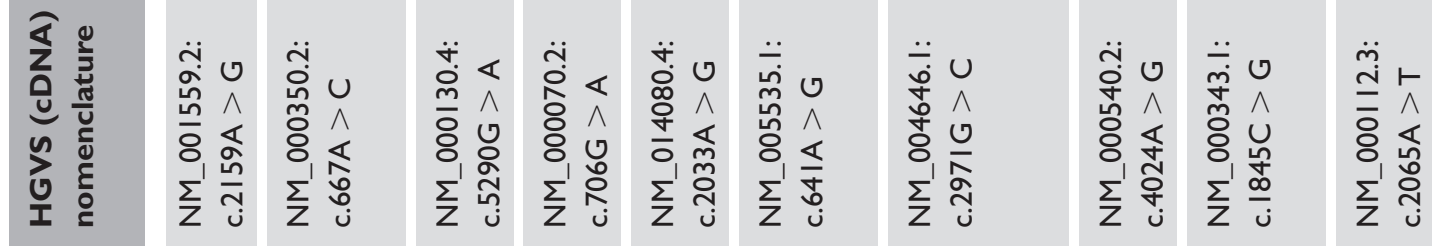

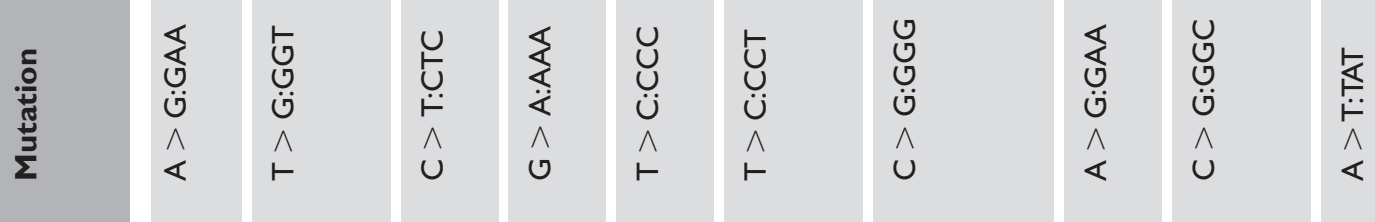

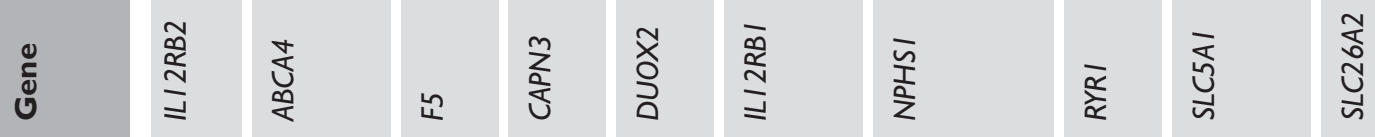

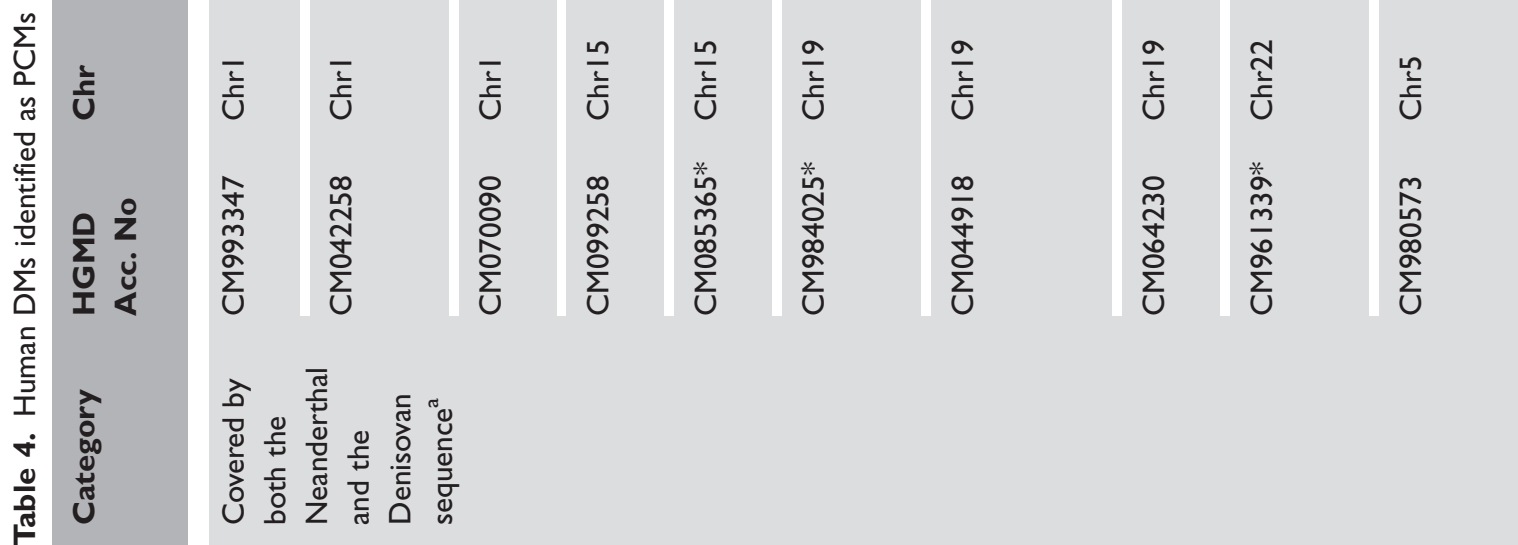




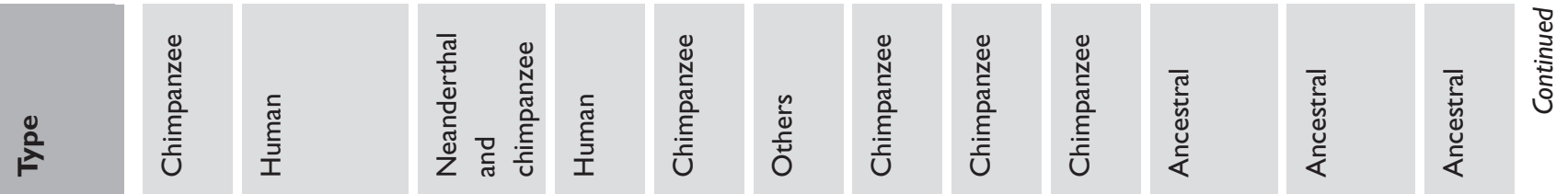

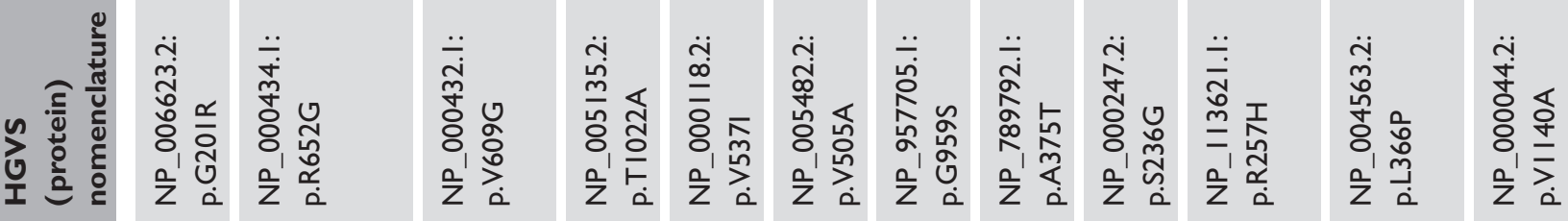

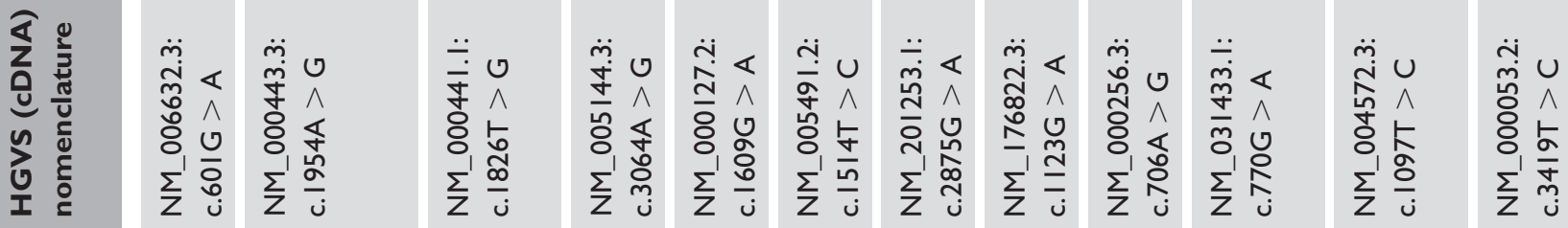

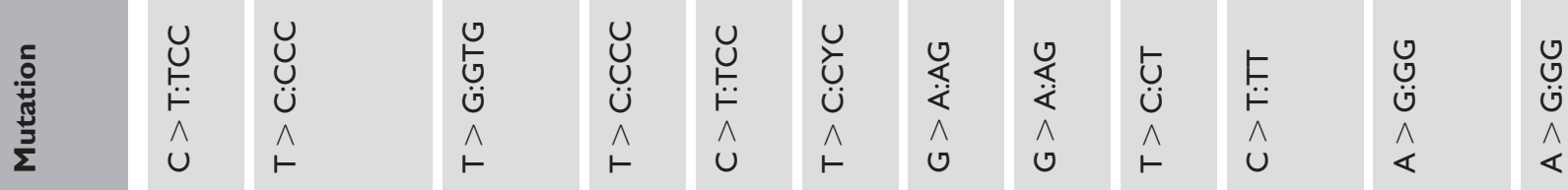

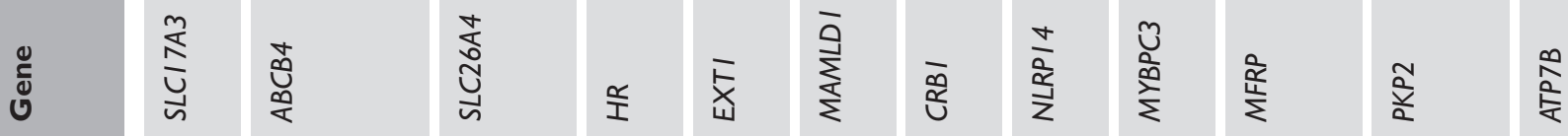

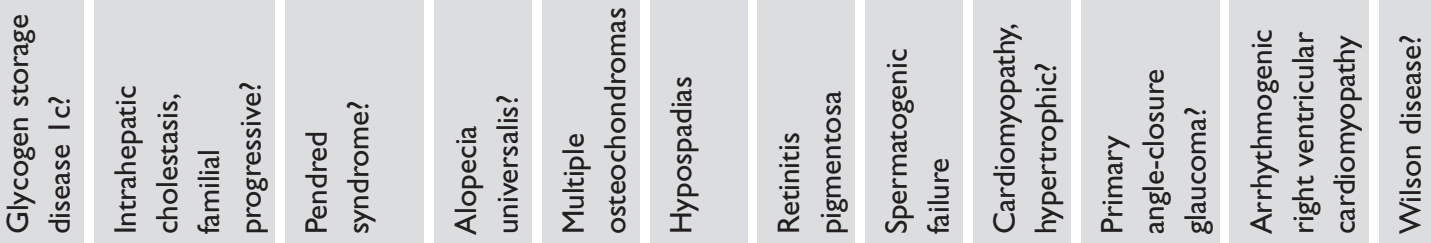

हैं

ยิ

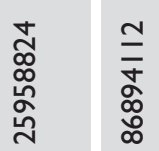

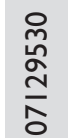

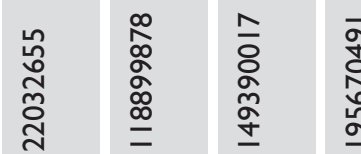

$\bar{\sigma}$
$\hat{0}$
0
$\stackrel{0}{\alpha}$

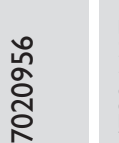

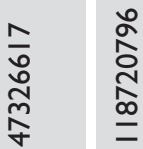

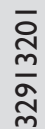

$\stackrel{\stackrel{n}{m}}{\frac{m}{\sigma}}$

ป

ปั

产

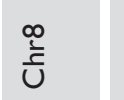

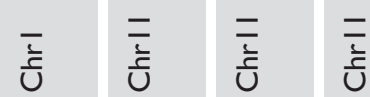

$\frac{m}{\frac{m}{U}} \frac{m}{\frac{1}{U}}$

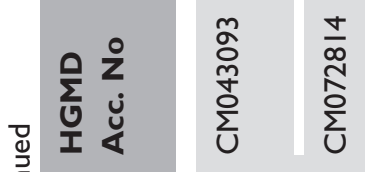

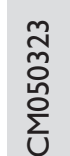

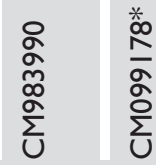

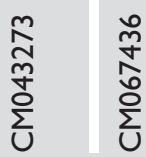

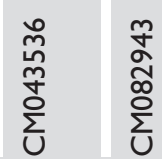

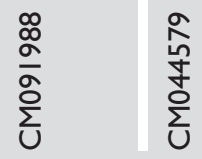

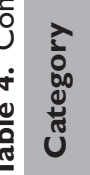

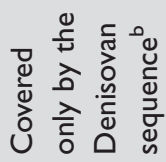




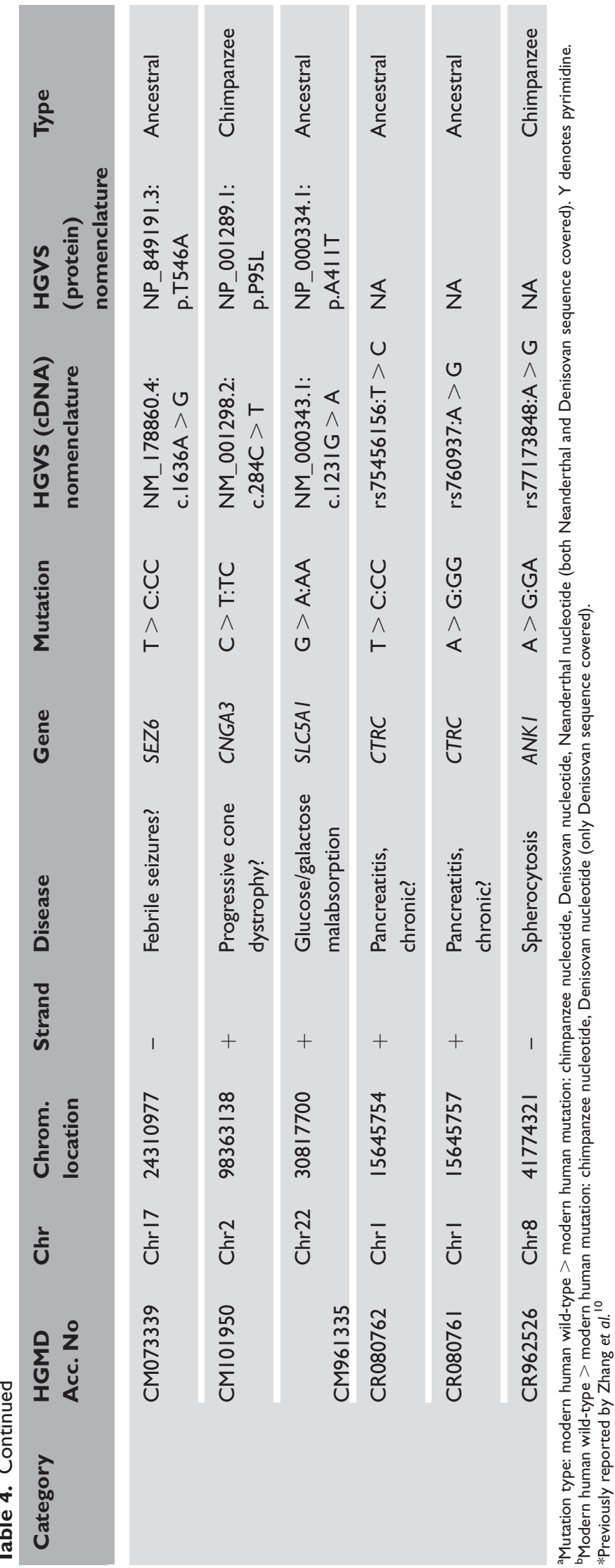

that was compensated only in the Neanderthal, one that was compensated only in the Denisovan, five that were compensated in both Neanderthal and Denisovan and 16 that were compensated only in the chimpanzee. There were also 18 mutations that differed between the Neanderthal and the Denisovan, which could imply that such mutations were identical-by-state (Tables 2 and 3 ).

\section{Disease-causing PCMs}

There were 16 human DMs that were found to be potentially compensated in the chimpanzee, Denisovan or Neanderthal (covered by both the Neanderthal and the Denisovan sequence) and 12 human DMs potentially compensated in the chimpanzee or Denisovan (covered only by the Denisovan sequence) (Table 4).

Of the human DMs that were potentially compensated in the chimpanzee, Denisovan or Neanderthal, only the putatively pathological F5 variant was specific to the Denisovan. In humans, this missense mutation, Val1736Met, is associated with activated protein $\mathrm{C}$ resistance and an increased risk of thromboembolism and recurrent miscarriage. ${ }^{20,21}$ It is unclear at this juncture whether this variant was indeed a PCM in the Denisovan or whether it could instead have been associated with disease in this archaic hominin.

Even though Denisovans appear to be more closely related to Neanderthals than humans, the Neanderthal and Denisovan were discrepant with respect to certain PCMs (eg the SLC5A1 H615Q variant associated with glucose-galactose malabsorption). In this case, the Denisovan (and the chimpanzee) possessed the allele that was mutant in humans (G), whereas the Neanderthal possessed the allele $(\mathrm{C})$ which was wild-type in humans. In this context, it may be pertinent to mention that SLC5A1 is located on chromosome 22q12.3 within a region of putative gene flow from Neanderthal to Eurasian. 1

Some of the PCMs listed in Table 4 may well have been misclassified by the original authors as disease-causing in human (especially those variants which have been allocated a '?' by the HGMD; see 
Table 4) when they were actually neutral polymorphisms; however, this is much less likely in the case of the 16 human disease-causing mutations that are covered by both the Neanderthal and Denisovan sequences. These mutant alleles would have had to have been maintained in both Neanderthal and Denisovan populations for $\sim 640,000$ years, when these two hominins last shared a common ancestor, and this would have been unlikely if such variants had been neutral polymorphisms.

Statistically enriched GO terms were identified for genes containing human DMs identified as PCMs (Table 4) against a background of known diseasecausing genes (from the HGMD) and are shown in Table S1. Five significantly enriched GO terms were found; all relate to the plasma membrane.

With respect to the DPs/FPs, 100 DPs, 39 FPs and 43 DFPs were covered by both the Neanderthal and Denisovan sequences (Table S2), while 52 DPs, 26 FPs and 27 DFPs were covered by the Denisovan but not the Neanderthal sequence (Table S3); these DPs/FPs may be relevant to human genetic disease.

\section{Human variants with significantly different population frequencies at sites of PCMs}

The $\mathrm{F}_{\mathrm{ST}}$ was used to quantify the allele frequency differences for the different polymorphic PCMs between extant African, Asian and European populations. Alleles that have been the target of localised positive selection tend to exhibit unusually high $\mathrm{F}_{\mathrm{ST}}$ values. $^{22,23} \mathrm{We}$ therefore compared the $\mathrm{F}_{\mathrm{ST}}$ values of the ancestral polymorphic PCMs with the empirical $\mathrm{F}_{\mathrm{ST}}$ distribution derived from all HapMap SNPs (International HapMap Consortium, 2007), ${ }^{24}$ to assess the significance of individual $\mathrm{F}_{\mathrm{ST}}$ values. We identified six PCMs with significantly elevated $\mathrm{F}_{\mathrm{ST}}$ values (Table 5).

Although four of these PCMs had already been identified in our previous comparative analysis of the human, chimpanzee and Neanderthal genomes, ${ }^{10}$ two novel PCMs were identified in the putative cation exchanger SLC24A5 (DP) gene and in the alcohol dehydrogenase $A D H 1 B(\mathrm{FP})$ gene. These genes have in common the GO terms

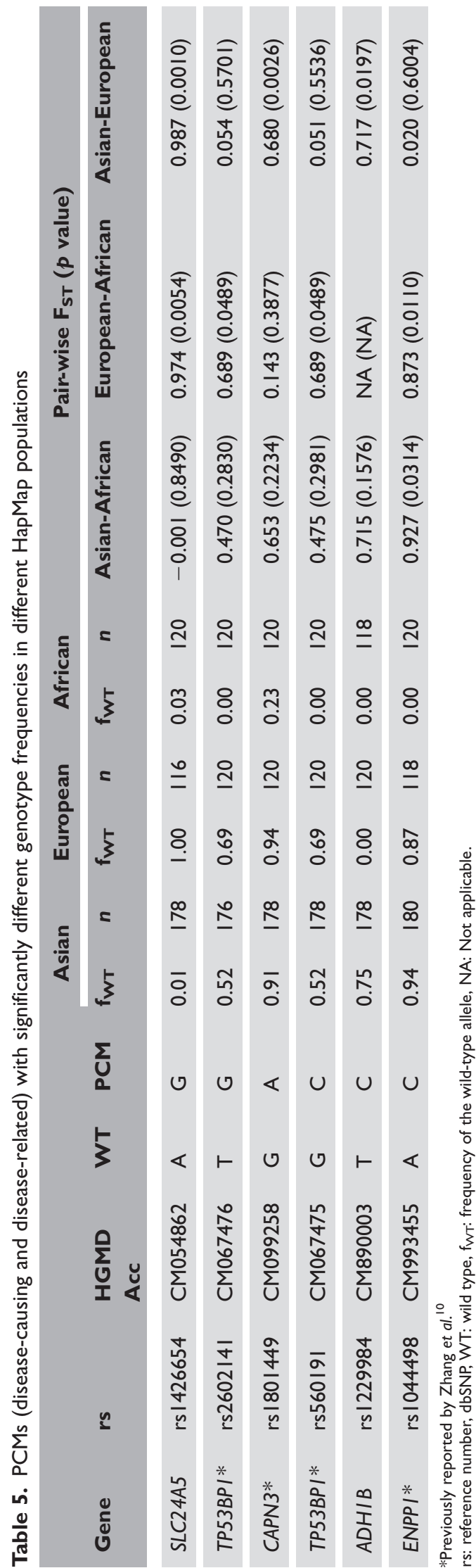


GO:0046872, GO:0043169 and GO:0043167, terms which relate to metal ion binding, cation binding and ion binding, respectively. The SLC24A5 variant appears to be associated with increased skin pigmentation and predominates in African/East Asian populations. ${ }^{25,26}$

In conclusion, using the newly reported genome sequence from a Denisovan hominin, we have identified a number of PCMs in the chimpanzee, Neanderthal and Denisovan. Those human PCMs that were ancestral (ie both the Denisovan nucleotide and the chimpanzee nucleotide were identical to the human DM/disease-associated mutation) could potentially be indicative of either the human lineage-specific loss of compensatory nucleotide changes within the respective genes carrying the PCM, or adaptive differences between modern humans and Denisovans.

\section{References}

1. Green, R.E., Krause, J., Briggs, A.W., Maricic, T. et al. (2010), 'A draft sequence of the Neanderthal genome', Science Vol. 328, pp. 710-722.

2. Noonan, J.P. (2010), 'Neanderthal genomics and the evolution of modern humans', Genome Res. Vol. 20, pp. 547-553.

3. Gibbons, A. (2010), 'Paleogenetics. Close encounters of the prehistoric kind', Science Vol. 328, pp. 680-684.

4. Reich, D., Green, R.E., Kircher, M., Krause, J. et al. (2010), 'Genetic history of an archaic hominin group from Denisova Cave in Siberia', Nature Vol. 468, pp. 1053-1060.

5. Reich, D., Green, R.E., Kircher, M., Krause, J. et al. (2010), 'The complete mitochondrial DNA genome of an unknown hominin from southern Siberia', Nature Vol. 468, pp. 1053-1060.

6. Krause, J., Fu, Q., Good, J.M., Viola, B. et al. (2010), 'The complete mitochondrial DNA genome of an unknown hominin from southern Siberia', Nature Vol. 464, pp. 894-897.

7. Martinón-Torres, M., Dennell, R. and Bermúdez de Castro, J.M. (2011), 'The Denisova hominin need not be an out of Africa story', J. Hum. Evol. Vol. 60, pp. 251-255.

8. Di Rienzo, A. and Hudson, R.R. (2005), 'An evolutionary framework for common diseases: The ancestral-susceptibility model', Trends Genet. Vol. 21, pp. 596-601.
9. Crespi, B.J. (2010), 'The origins and evolution of genetic disease risk in modern humans', Ann. N. Y. Acad. Sci. Vol. 1206, pp. 80-109.

10. Zhang, G., Pei, Z., Krawczak, M., Ball, E.V. et al. (2010), 'Triangulation of the human, chimpanzee, and Neanderthal genome sequences identifies potentially compensated mutations', Hum. Mutat. Vol. 31, pp. $1286-1293$.

11. Gao, L. and Zhang, J. (2003), 'Why are some human disease-associated mutations fixed in mice?', Trends Genet. Vol. 19. pp. 678-681.

12. Azevedo, L., Suriano, G., van Asch, B., Harding, R.M. and Amorim, A. (2006), 'Epistatic interactions: How strong in disease and evolution?', Trends Genet. Vol. 22, pp. 581-585.

13. Ferrer-Costa, C., Orozco, M. and de la Cruz, X. (2007), 'Characterization of compensated mutations in terms of structural and physico-chemical properties'. J. Mol. Biol. Vol. 365, pp. 249-256.

14. Corona, E., Dudley, J.T. and Butte, A.J. (2010), 'Extreme evolutionary disparities seen in positive selection across seven complex diseases', PLoS One Vol. 5, p. e12236.

15. Baresić, A., Hopcroft, L.E., Rogers, H.H., Hurst, J.M. et al. (2010), 'Compensated pathogenic deviations: Analysis of structural effects', J. Mol. Biol. Vol. 396, pp. 19-30.

16. Stenson, P.D., Mort, M., Ball, E.V., Howells, K. et al. (2009), 'The Human Gene Mutation Database: 2008 update', Genome Med. Vol. 1, p. 13.

17. Huang da, W., Sherman, B.T. and Lempicki, R.A. (2009), 'Systematic and integrative analysis of large gene lists using DAVID bioinformatics resources', Nat. Protoc. Vol. 4, pp. 44-57.

18. Benjamini, Y. and Hochberg, Y. (1995), 'Controlling the false discovery rate: A practical and powerful approach to multiple testing', J. R. Stat. Soc. Series B Vol. 57, pp. 289-300.

19. Weir, B.S. and Hill, W.G. (2002), 'Estimating F-statistics', Annu. Rev, Genet. Vol. 36, pp. 721-750.

20. Dawood, F, Mountford, R., Farquharson, R. and Quenby, S. (2007), 'Genetic polymorphisms on the factor $\mathrm{V}$ gene in women with recurrent miscarriage and acquired APCR'. Hum. Reprod. Vol. 22, pp. 2546-2553.

21. Chegeni, R., Kazemi, B., Hajifathali, A., Pourfathollah, A. et al. (2007), 'Factor V mutations in Iranian patients with activated protein C resistance and venous thrombosis', Thromb. Res. Vol. 119, pp. 189-193.

22. Holsinger, K.E. and Weir, B.S. (2009), 'Genetics in geographically structured populations: Defining, estimating and interpreting $\mathrm{F}_{\mathrm{ST}}$ ', Nat. Rev. Genet. Vol. 10, pp. 639-650.

23. Thornton, K.R. and Jensen, J.D. (2007), 'Controlling the false-positive rate in multilocus genome scans for selection', Genetics Vol. 175, pp. $737-750$.

24. International HapMap Consortium, Frazer, K.A., Ballinger, D.G., Cox, D.R. et al. (2007), 'A second generation human haplotype map of over 3.1 million SNPs'. Nature Vol. 449, pp. 851-861.

25. Lamason, R.L., Mohideen, M.A., Mest, J.R., Wong, A.C. et al. (2005), 'SLC24A5, a putative cation exchanger, affects pigmentation in zebrafish and humans', Science Vol. 310, pp. 1782-1786.

26. Stokowski, R.P., Pant, P.V., Dadd, T., Fereday, A. et al. (2007), 'A genomewide association study of skin pigmentation in a South Asian population', Am. J. Hum. Genet. Vol. 81, pp. 1119-1132. 


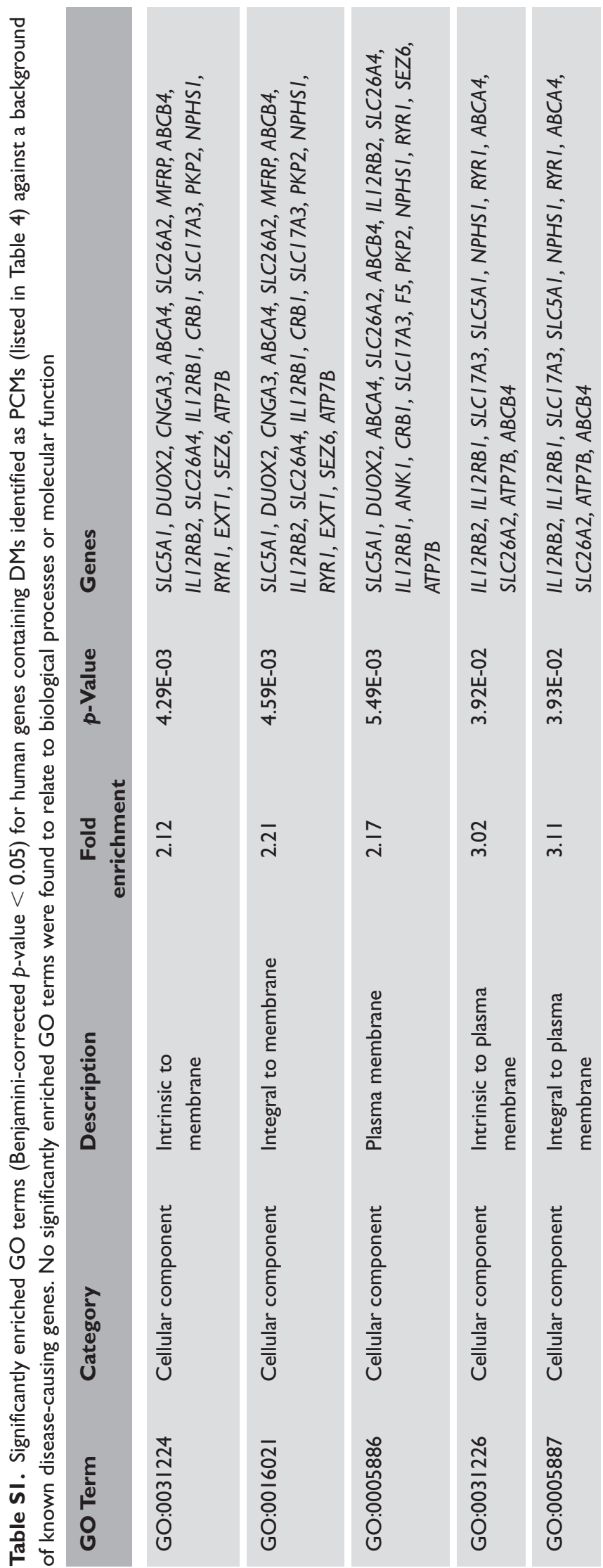


Table S2. PCMs covered by both the Denisovan sequence and the Neanderthal sequence

\begin{tabular}{|c|c|c|c|c|c|c|c|c|c|}
\hline $\begin{array}{l}\text { HGMD } \\
\text { Acc }\end{array}$ & Chr & Location & Strand & Tag & Disease & Gene & Mutation & AA seq & Type \\
\hline CM031993 & Chrl & 9246497 & + & DFP & $\begin{array}{l}\text { Cortisone reductase } \\
\text { deficiency, partial }\end{array}$ & H6PD & $\mathrm{G}>\mathrm{A}: \mathrm{AAA}$ & Arg-Gln & Human \\
\hline CM040788 & Chrl & II 828655 & - & DP & $\begin{array}{l}\text { Stroke, increased } \\
\text { risk, association with }\end{array}$ & NPPA & $A>G: G G G$ & Term-Arg & Human \\
\hline CMI006II & Chrl & $120055 \mid 3$ & + & DFP & $\begin{array}{l}\text { Breast cancer, } \\
\text { reduced risk, } \\
\text { association with }\end{array}$ & MIIP & A $>$ G:GGG & Lys-Glu & Human \\
\hline CM980072 & Chrl & 21767322 & + & DFP & $\begin{array}{l}\text { Hypophosphatasia, } \\
\text { association with }\end{array}$ & $A L P L$ & $\mathrm{~T}>\mathrm{C}: \mathrm{CCC}$ & Tyr-His & Human \\
\hline CM056598 & Chrl & 31865112 & + & DP & $\begin{array}{l}\text { Polydipsia- } \\
\text { hyponatraemia, } \\
\text { association with }\end{array}$ & HCRTRI & $\mathrm{A}>\mathrm{G}: \mathrm{GAA}$ & Ile-Val & Chimpanzee \\
\hline CM994I22 & Chrl & 35033356 & + & DFP & $\begin{array}{l}\text { Atherosclerosis, } \\
\text { association with }\end{array}$ & GJA4 & $\mathrm{C}>\mathrm{T}: \mathrm{TTT}$ & Pro-Ser & Human \\
\hline CM0655I4 & Chrl & 55410663 & - & DP & $\begin{array}{l}\text { Parkinson's disease, } \\
\text { risk, association with }\end{array}$ & USP24 & $\mathrm{G}>\mathrm{A}: \mathrm{AAA}$ & Thr-lle & Human \\
\hline CM073I4I & Chrl & 67457975 & + & DP & $\begin{array}{l}\text { Psoriasis, increased } \\
\text { risk, association with }\end{array}$ & IL23R & $\mathrm{T}>\mathrm{C}: \mathrm{CCC}$ & Leu-Pro & Human \\
\hline CM993347 & Chrl & 67633930 & + & DM & Atopy & ILI $2 R B 2$ & $\mathrm{~A}>\mathrm{G}: \mathrm{GAA}$ & His-Arg & Chimpanzee \\
\hline CM067986 & Chrl & 86873963 & + & DP & $\begin{array}{l}\text { Chloride channel } \\
\text { deficiency, } \\
\text { association with }\end{array}$ & CLCA3P & $\mathrm{C}>\mathrm{G}: \mathrm{GGG}$ & Tyr-Term & Human \\
\hline CM042258 & Chrl & 94337039 & - & DM & Stargardt disease & $A B C A 4$ & $\mathrm{~T}>\mathrm{G}: \mathrm{GGT}$ & Lys-Gln & $\begin{array}{l}\text { Denisova } \\
\text { and } \\
\text { chimpanzee }\end{array}$ \\
\hline CM067656 & Chrl & $15649 \mid 643$ & + & DP & $\begin{array}{l}\text { Guillain-Barré } \\
\text { syndrome, reduced } \\
\text { risk, association } \\
\text { with? }\end{array}$ & $C D / A$ & $\mathrm{C}>\mathrm{G}: \mathrm{GGC}$ & Cys-Trp & $\begin{array}{l}\text { Denisova } \\
\text { and } \\
\text { chimpanzee }\end{array}$ \\
\hline CM070090 & Chrl & 167765599 & - & DM & Thrombosis? & F5 & $\mathrm{C}>\mathrm{T}: \mathrm{CTC}$ & Val-Met & Denisovan \\
\hline CM099896 & Chrl & $1736 \mid 5346$ & - & DP & $\begin{array}{l}\text { Schizophrenia, } \\
\text { association with }\end{array}$ & $T N R$ & C > T:TTT & Arg-Lys & Human \\
\hline CM023569 & Chrl & 199313698 & - & DP & $\begin{array}{l}\text { Hypokalaemic } \\
\text { periodic paralysis, } \\
\text { association with? }\end{array}$ & CACNAIS & $\mathrm{G}>\mathrm{A}: \mathrm{GRA}$ & Gly-Gly & Unsure \\
\hline CM9200I0 & Chrl & 228912417 & - & DP & $\begin{array}{l}\text { Hypertension, } \\
\text { association with }\end{array}$ & AGT & $A>G: G G G$ & Met-Thr & Human \\
\hline
\end{tabular}


Table S2. Continued

\begin{tabular}{|c|c|c|c|c|c|c|c|c|c|}
\hline $\begin{array}{l}\text { HGMD } \\
\text { Acc }\end{array}$ & Chr & Location & Strand & Tag & Disease & Gene & Mutation & AA seq & Type \\
\hline CM065I55 & Chrl & 240108924 & + & DP & $\begin{array}{l}\text { Colorectal cancer, } \\
\text { increased risk, } \\
\text { association with }\end{array}$ & EXOI & $\mathrm{G}>\mathrm{A}: \mathrm{AAA}$ & Glu-Lys & Human \\
\hline CM033447 & ChrlO & 42926693 & + & DP & $\begin{array}{l}\text { Hirschsprung } \\
\text { disease, association } \\
\text { with }\end{array}$ & RET & $A>G: G G G$ & Ala-Ala & Human \\
\hline CM068I90 & ChrlO & 54198272 & - & FP & $\begin{array}{l}\text { Increased serum } \\
\text { mannose-binding } \\
\text { lectin (MBL) level, } \\
\text { association with? }\end{array}$ & MBL2 & $C>G: C G G$ & Leu-Leu & Ancient \\
\hline CM033482 & ChrlO & 64085190 & + & DP & $\begin{array}{l}\text { Uric acid } \\
\text { nephrolithiasis, } \\
\text { association with }\end{array}$ & znf365d & $\mathrm{G}>\mathrm{A}: \mathrm{GGA}$ & Ala-Thr & Neanderthal \\
\hline CM06746I & ChrlO & 81691702 & - & DP & $\begin{array}{l}\text { Lung cancer, } \\
\text { susceptibility to, } \\
\text { association with }\end{array}$ & SFTPD & $\mathrm{T}>\mathrm{C}: \mathrm{CCC}$ & Thr-Ala & Human \\
\hline CM035804 & Chrll & 524242 & - & DP & $\begin{array}{l}\text { Bladder cancer, } \\
\text { association with? }\end{array}$ & HRAS & $A>G: G A G$ & His-His & $\begin{array}{l}\text { Neanderthal } \\
\text { and } \\
\text { chimpanzee }\end{array}$ \\
\hline CM02589I & Chrll & 74585230 & + & $\mathrm{FP}$ & $\begin{array}{l}\text { Decreased enzyme } \\
\text { activity, association } \\
\text { with }\end{array}$ & SLCO2BI & $\mathrm{C}>\mathrm{T}: \mathrm{TTT}$ & Ser-Phe & Human \\
\hline CM0804I5 & Chrll & 113308238 & + & $\mathrm{FP}$ & $\begin{array}{l}\text { Altered receptor } \\
\text { function, association } \\
\text { with }\end{array}$ & HTR3B & $A>C: C C C$ & Tyr-Ser & Human \\
\hline CM950862 & Chrl2 & 5473868 & + & DP & $\begin{array}{l}\text { Schizophrenia, } \\
\text { severe, increased } \\
\text { risk, association with }\end{array}$ & NTF3 & $G>A: A G G$ & Gly-Glu & Chimpanzee \\
\hline CM093840 & Chrl2 & 6023795 & - & DP & $\begin{array}{l}\text { von Willebrand } \\
\text { disease, quantitative } \\
\text { type, association } \\
\text { with }\end{array}$ & VWF & $\mathrm{T}>\mathrm{C}: \mathrm{CCC}$ & Thr-Ala & Human \\
\hline CM994637 & $\mathrm{Chr} / 2$ & 6327323 & - & DFP & $\begin{array}{l}\text { Hypertension, } \\
\text { reduced risk, } \\
\text { association with }\end{array}$ & SCNNIA & $\mathrm{T}>\mathrm{C}: \mathrm{CCC}$ & Thr-Ala & Human \\
\hline CM00367I & Chrl2 & 14884706 & - & $\mathrm{FP}$ & $\begin{array}{l}\text { Dombrock blood } \\
\text { group variation }\end{array}$ & ART4 & $\mathrm{T}>\mathrm{C}: \mathrm{TCC}$ & Asn-Asp & Ancient \\
\hline CM077900 & Chrl2 & 70659129 & + & $\mathrm{FP}$ & $\begin{array}{l}\text { Increased mRNA } \\
\text { expression, } \\
\text { association with? }\end{array}$ & TPH2 & $\mathrm{G}>\mathrm{A}: \mathrm{GAA}$ & Pro-Pro & Ancient \\
\hline
\end{tabular}


Table S2. Continued

\begin{tabular}{|c|c|c|c|c|c|c|c|c|c|}
\hline $\begin{array}{l}\text { HGMD } \\
\text { Acc }\end{array}$ & Chr & Location & Strand & Tag & Disease & Gene & Mutation & AA seq & Type \\
\hline CM085048 & Chrl2 & 78539038 & - & DP & $\begin{array}{l}\text { Schizophrenia in } \\
\text { females, association } \\
\text { with }\end{array}$ & PAWR & $A>C: C C C$ & Ile-Met & Human \\
\hline CM033453 & $\mathrm{Chr} / 2$ & 107542027 & - & DFP & $\begin{array}{l}\text { Coronary heart } \\
\text { disease, decreased } \\
\text { risk, in African } \\
\text { Americans, } \\
\text { association with }\end{array}$ & SELPLG & $\mathrm{C}>\mathrm{T}: \mathrm{TTT}$ & Met-Ile & Human \\
\hline CM022034 & Chrl3 & 32526193 & + & DP & $\begin{array}{l}\text { Age-related } \\
\text { phenotypes, } \\
\text { association with }\end{array}$ & $K L$ & $\mathrm{G}>\mathrm{C}: \mathrm{CGG}$ & Cys-Ser & Chimpanzee \\
\hline CM033777 & Chrl4 & 24170122 & - & DP & $\begin{array}{l}\text { Apoptosis, unable to } \\
\text { induce, association } \\
\text { with }\end{array}$ & GZMB & $A>G: G G G$ & Tyr-His & Human \\
\hline CM070246 & Chrl4 & 60993992 & + & DFP & $\begin{array}{l}\text { Cerebral infarction, } \\
\text { association with }\end{array}$ & PRKCH & $\mathrm{G}>\mathrm{A}: \mathrm{AAA}$ & Val-Ile & Human \\
\hline CM067476 & ChrI5 & 41511938 & - & DP & $\begin{array}{l}\text { Lung cancer, } \\
\text { susceptibility to, } \\
\text { association with }\end{array}$ & TP53ВPI & $\mathrm{T}>\mathrm{G}: \mathrm{GGG}$ & Lys-Gln & Human \\
\hline CM067475 & Chrl5 & 41555066 & - & DP & $\begin{array}{l}\text { Lung cancer, } \\
\text { susceptibility to, } \\
\text { association with }\end{array}$ & TP53ВPI & $\mathrm{G}>\mathrm{C}: \mathrm{CCC}$ & Asp-Glu & Human \\
\hline CM085365 & ChrI5 & 43185730 & - & DM & Hypothyroidism & DUOX2 & $\mathrm{T}>\mathrm{C}: \mathrm{CCC}$ & His-Arg & Human \\
\hline CM054862 & ChrI5 & 46213776 & + & DP & $\begin{array}{l}\text { Increased skin } \\
\text { pigmentation, } \\
\text { association with }\end{array}$ & SLC24A5 & $A>G: G G G$ & Thr-Ala & Human \\
\hline CM057869 & Chrl5 & 76704628 & - & $\mathrm{FP}$ & $\begin{array}{l}\text { Altered function, } \\
\text { association with }\end{array}$ & CHRNB4 & $\mathrm{T}>\mathrm{C}: \mathrm{CTT}$ & Met-Val & Chimpanzee \\
\hline CM03I698 & Chrl5 & 97295748 & + & DP & $\begin{array}{l}\text { Increased longevity, } \\
\text { association with? }\end{array}$ & $I G F I R$ & $G>A: A G G$ & Glu-Glu & Chimpanzee \\
\hline CM057585 & Chrl6 & |442858 & - & DP & $\begin{array}{l}\text { Lower femoral neck } \\
\text { bone mineral density } \\
\text { in women, } \\
\text { association with }\end{array}$ & CLCN7 & $\mathrm{C}>\mathrm{T}: \mathrm{TCT}$ & Val-Met & $\begin{array}{l}\text { Neanderthal } \\
\text { and } \\
\text { chimpanzee }\end{array}$ \\
\hline CM983400 & Chrl6 & 27263704 & + & DFP & $\begin{array}{l}\text { Asthma, atopic, } \\
\text { association with }\end{array}$ & IL4R & $A>G: G G G$ & Ile-Val & Human \\
\hline CM067985 & Chrl6 & 87788983 & + & DP & $\begin{array}{l}\text { Cadherin deficiency, } \\
\text { association with }\end{array}$ & CDHI5 & $C>A: A A A$ & Tyr-Term & Human \\
\hline
\end{tabular}


Table S2. Continued

\begin{tabular}{|c|c|c|c|c|c|c|c|c|c|}
\hline $\begin{array}{l}\text { HGMD } \\
\text { Acc }\end{array}$ & Chr & Location & Strand & Tag & Disease & Gene & Mutation & AA seq & Type \\
\hline CM057933 & Chrl7 & 4585312 & - & DP & $\begin{array}{l}\text { Atherosclerotic } \\
\text { stenosis, increased, } \\
\text { association with }\end{array}$ & CXCLI6 & $G>A: A A G$ & Ala-Val & $\begin{array}{l}\text { Denisova } \\
\text { and } \\
\text { chimpanzee }\end{array}$ \\
\hline CM077855 & Chrl7 & 7532893 & + & DP & $\begin{array}{l}\text { Breast cancer, } \\
\text { oestrogen receptor } \\
\text { (ER) negative, } \\
\text { association with? }\end{array}$ & WRAP53 & $C>G: G G G$ & Arg-Gly & Human \\
\hline CM08738I & Chrl7 & 7987497 & - & $\mathrm{FP}$ & $\begin{array}{l}\text { Increased sex } \\
\text { hormone-binding } \\
\text { globulin levels, } \\
\text { association with }\end{array}$ & PERI & $\mathrm{C}>\mathrm{G}: \mathrm{GGG}$ & Ala-Pro & Human \\
\hline CM067489 & Chrl7 & 16468520 & - & DP & $\begin{array}{l}\text { Lung cancer, } \\
\text { susceptibility to, } \\
\text { association with }\end{array}$ & ZNF624 & $C>A: A A A$ & Lys-Asn & Human \\
\hline СМ030773 & Chrl7 & 19753133 & - & DP & $\begin{array}{l}\text { Cardiac disease, } \\
\text { susceptibility to, } \\
\text { association with }\end{array}$ & AKAPIO & $\mathrm{T}>\mathrm{C}: \mathrm{CCC}$ & Ile-Val & Human \\
\hline CM067336 & Chrl7 & 19802050 & - & DP & $\begin{array}{l}\text { Lung cancer, } \\
\text { susceptibility to, } \\
\text { association with }\end{array}$ & AKAPIO & C $>$ T:TTT & Arg-His & Human \\
\hline CM0963I5 & Chrl7 & 38498462 & - & DFP & $\begin{array}{l}\text { Cervical cancer, } \\
\text { decreased risk, } \\
\text { association with }\end{array}$ & BRCAI & $\mathrm{G}>\mathrm{A}: \mathrm{AAA}$ & Pro-Leu & Human \\
\hline CM0934I8 & Chrl7 & 39581073 & + & DP & $\begin{array}{l}\text { Hip bone mineral } \\
\text { density, association } \\
\text { with? }\end{array}$ & CI7orf53 & $A>C: C C C$ & Thr-Pro & Human \\
\hline CM032397 & Chrl7 & 41432502 & + & DP & $\begin{array}{l}\text { Progressive } \\
\text { supranuclear palsy, } \\
\text { association with }\end{array}$ & STH & $A>G: G A A$ & Gln-Arg & Chimpanzee \\
\hline CM064363 & Chrl7 & 45788957 & + & DP & $\begin{array}{l}\text { Organ involvement } \\
\text { in pseudoxantnoma } \\
\text { elasticum (PXE), } \\
\text { association with }\end{array}$ & XYLT2 & $\mathrm{T}>\mathrm{C}: \mathrm{CCC}$ & Tyr-Tyr & Human \\
\hline CM092499 & Chrl7 & 76468818 & + & $\mathrm{FP}$ & $\begin{array}{l}\text { Altered splicing, } \\
\text { association with? }\end{array}$ & KIAA/303 & $\mathrm{A}>\mathrm{G}: \mathrm{GAA}$ & Gln-Gln & Chimpanzee \\
\hline CM08043I & Chrl9 & 11091881 & + & $\mathrm{FP}$ & $\begin{array}{l}\text { Increased plasma } \\
\text { low-density } \\
\text { lipoprotein } \\
\text { cholesterol, } \\
\text { association with }\end{array}$ & LDLR & $\mathrm{T}>\mathrm{C}: \mathrm{CTT}$ & Val-Val & Chimpanzee \\
\hline
\end{tabular}


Table S2. Continued

\begin{tabular}{|c|c|c|c|c|c|c|c|c|c|}
\hline $\begin{array}{l}\text { HGMD } \\
\text { Acc }\end{array}$ & Chr & Location & Strand & Tag & Disease & Gene & Mutation & AA seq & Type \\
\hline CM984025 & Chrl9 & 18047618 & - & DM & $\begin{array}{l}\text { Mycobacterial } \\
\text { infection }\end{array}$ & $|L| 2 R B \mid$ & $\mathrm{T}>\mathrm{C}: \mathrm{CCT}$ & Gln-Arg & $\begin{array}{l}\text { Denisova } \\
\text { and } \\
\text { chimpanzee }\end{array}$ \\
\hline CM0449I8 & Chrl9 & 41022117 & - & DM & $\begin{array}{l}\text { Congenital nephrotic } \\
\text { syndrome, Finnish } \\
\text { type }\end{array}$ & NPHSI & $C>G: G G G$ & Val-Leu & Human \\
\hline CM073386 & Chrl9 & 50087554 & + & DP & $\begin{array}{l}\text { Alzheimer's disease, } \\
\text { late-onset, } \\
\text { association with? }\end{array}$ & TOMM40 & $\mathrm{T}>\mathrm{C}: \mathrm{CCC}$ & Phe-Phe & Human \\
\hline CM0048I4 & Chrl9 & 50546759 & - & DFP & $\begin{array}{l}\text { Basal cell carcinoma, } \\
\text { reduced risk, } \\
\text { association with }\end{array}$ & $E R C C 2$ & $\mathrm{~T}>\mathrm{G}: \mathrm{GGG}$ & Lys-Gln & Human \\
\hline CM096319 & Chr2 & II27657| & - & DP & $\begin{array}{l}\text { Chronic kidney } \\
\text { disease in individuals } \\
\text { with low } \\
\text { triglycerides, } \\
\text { association with }\end{array}$ & ROCK2 & $\mathrm{G}>\mathrm{T}: \mathrm{TGT}$ & Thr-Asn & $\begin{array}{l}\text { Neanderthal } \\
\text { and } \\
\text { chimpanzee }\end{array}$ \\
\hline CM052876 & Chr2 & 49043425 & - & DP & $\begin{array}{l}\text { Menstrual cycle } \\
\text { length, association } \\
\text { with }\end{array}$ & FSHR & $\mathrm{C}>\mathrm{T}: \mathrm{TTT}$ & Ser-Asn & Human \\
\hline CM073086 & Chr2 & 85634047 & - & DP & $\begin{array}{l}\text { Higher body mass } \\
\text { index, association } \\
\text { with }\end{array}$ & GGCX & $\mathrm{C}>\mathrm{T}: \mathrm{TCC}$ & Arg-Gln & Chimpanzee \\
\hline CM087379 & Chr2 & 100957736 & + & FP & $\begin{array}{l}\text { Higher testosterone } \\
\text { levels, association } \\
\text { with }\end{array}$ & NPAS2 & $A>G: G G G$ & Thr-Ala & Human \\
\hline CM004559 & Chr2 & 227369287 & - & DP & $\begin{array}{l}\text { Diabetes, type } 2 \text {, } \\
\text { association with }\end{array}$ & IRSI & $\mathrm{T}>\mathrm{C}: \mathrm{CCT}$ & Ala-Ala & $\begin{array}{l}\text { Denisova } \\
\text { and } \\
\text { chimpanzee }\end{array}$ \\
\hline CM085I46 & Chr2 & $2278394 \mid 3$ & + & DP & $\begin{array}{l}\text { Chronic obstructive } \\
\text { pulmonary disease, } \\
\text { association with }\end{array}$ & COL4A3 & $\mathrm{A}>\mathrm{G}: \mathrm{GAA}$ & His-Arg & Chimpanzee \\
\hline CMOI 4824 & Chr20 & 4653718 & + & DP & $\begin{array}{l}\text { Creutzfeldt-Jakob } \\
\text { disease, association } \\
\text { with }\end{array}$ & PRND & $\mathrm{C}>\mathrm{T}: \mathrm{TCT}$ & Thr-Met & $\begin{array}{l}\text { Neanderthal } \\
\text { and } \\
\text { chimpanzee }\end{array}$ \\
\hline CM064I2I & Chr20 & 44075813 & + & DP & $\begin{array}{l}\text { Leukaemia, risk, } \\
\text { association with }\end{array}$ & MMP9 & $\mathrm{G}>\mathrm{C}: \mathrm{CCC}$ & Arg-Pro & Human \\
\hline CM035699 & Chr2I & 14403236 & - & FP & $\begin{array}{l}\text { Plasma high-density } \\
\text { lipoprotein (HDL) } \\
\text { cholesterol, } \\
\text { association with }\end{array}$ & LIPI & $\mathrm{G}>\mathrm{T}: \mathrm{TTT}$ & Asp-Glu & Human \\
\hline
\end{tabular}


Table S2. Continued

\begin{tabular}{|c|c|c|c|c|c|c|c|c|c|}
\hline $\begin{array}{l}\text { HGMD } \\
\text { Acc }\end{array}$ & Chr & Location & Strand & Tag & Disease & Gene & Mutation & AA seq & Type \\
\hline CM0577II & Chr2I & 33536125 & + & DP & $\begin{array}{l}\text { Multiple sclerosis, } \\
\text { susceptibility to, } \\
\text { association with }\end{array}$ & IFNAR2 & $\mathrm{T}>\mathrm{G}: \mathrm{GGG}$ & Phe-Val & Human \\
\hline CM025479 & Chr2I & 44534334 & + & DP & $\begin{array}{l}\text { Alopecia universalis, } \\
\text { association with }\end{array}$ & AIRE & $\mathrm{C}>\mathrm{G}: \mathrm{GGG}$ & Ser-Arg & Human \\
\hline CM057927 & Chr22 & 21957369 & + & DP & $\begin{array}{l}\text { Bipolar disorder, } \\
\text { association with? }\end{array}$ & $B C R$ & $A>G: G G G$ & Asn-Ser & Human \\
\hline CM065332 & Chr22 & 24489289 & + & DP & $\begin{array}{l}\text { Colorectal cancer, } \\
\text { increased risk, } \\
\text { association with }\end{array}$ & MYOI8B & $\mathrm{G}>\mathrm{A}: \mathrm{AAA}$ & Gly-Glu & Human \\
\hline CM961339 & Chr22 & 30836050 & + & DM & $\begin{array}{l}\text { Glucose/galactose } \\
\text { malabsorption }\end{array}$ & SLC5AI & $C>$ G:GGC & His-Gln & $\begin{array}{l}\text { Denisova } \\
\text { and } \\
\text { chimpanzee }\end{array}$ \\
\hline CM096696 & Chr22 & 35792882 & - & DP & $\begin{array}{l}\text { Iron status and } \\
\text { erythrocyte volume, } \\
\text { association with }\end{array}$ & TMPRSS6 & A $>$ G:GGG & Val-Ala & Human \\
\hline CM092918 & Chr22 & 37827350 & + & $\mathrm{FP}$ & $\begin{array}{l}\text { Increased } \\
\text { antiretroviral activity, } \\
\text { association with }\end{array}$ & APOBEC $3 \mathrm{H}$ & $\mathrm{G}>\mathrm{C}: \mathrm{CCC}$ & Gly-Arg & Human \\
\hline CM910052 & Chr22 & 49410905 & - & DP & $\begin{array}{l}\text { Phenotype modifier, } \\
\text { association with? }\end{array}$ & ARSA & $\mathrm{G}>\mathrm{C}: \mathrm{CCC}$ & Thr-Ser & Human \\
\hline CM023348 & Chr3 & 336508 & + & DP & $\begin{array}{l}\text { Schizophrenia, } \\
\text { association with }\end{array}$ & $\mathrm{CHLI}$ & $\mathrm{C}>\mathrm{T}: \mathrm{TTT}$ & Leu-Phe & Human \\
\hline CM096382 & Chr3 & 46476217 & - & DFP & $\begin{array}{l}\text { Periodontitis, } \\
\text { aggressive, } \\
\text { association with }\end{array}$ & LTF & $\mathrm{T}>\mathrm{C}: \mathrm{CCC}$ & Lys-Arg & Human \\
\hline CM06658I & Chr3 & $1261097 \mid 4$ & - & DP & $\begin{array}{l}\text { Ulcerative colitis, } \\
\text { association with }\end{array}$ & MUCI 3 & $\mathrm{~T}>\mathrm{G}: \mathrm{GGG}$ & Arg-Ser & Human \\
\hline CM94I 277 & Chr3 & $1722 \mid 4994$ & - & DP & $\begin{array}{l}\text { Diabetes, type } 2 \text {, } \\
\text { association with }\end{array}$ & $S L C 2 A 2$ & $\mathrm{G}>\mathrm{A}: \mathrm{AAG}$ & Thr-Ile & $\begin{array}{l}\text { Denisova } \\
\text { and } \\
\text { chimpanzee }\end{array}$ \\
\hline CM065290 & Chr3 & $1879257 \mid 2$ & + & DP & $\begin{array}{l}\text { Nephropathy, } \\
\text { reduced risk, } \\
\text { association with }\end{array}$ & KNGI & $\mathrm{T}>\mathrm{C}: \mathrm{CCC}$ & Met-Thr & Human \\
\hline CM025429 & Chr4 & 2960297 & + & FP & $\begin{array}{l}\text { Increased enzymatic } \\
\text { activity, association } \\
\text { with }\end{array}$ & GRK4 & G > T:TTT & Arg-Leu & Human \\
\hline CM094340 & Chr4 & 38476105 & - & DFP & $\begin{array}{l}\text { Leprosy, association } \\
\text { with }\end{array}$ & $T L R I$ & $\mathrm{~T}>\mathrm{C}: \mathrm{CCC}$ & Asn-Ser & Human \\
\hline
\end{tabular}


Table S2. Continued

\begin{tabular}{|c|c|c|c|c|c|c|c|c|c|}
\hline $\begin{array}{l}\text { HGMD } \\
\text { Acc }\end{array}$ & Chr & Location & Strand & Tag & Disease & Gene & Mutation & AA seq & Type \\
\hline CM890003 & Chr4 & 100458342 & - & $\mathrm{FP}$ & $\begin{array}{l}\text { Alcohol } \\
\text { dehydrogenase beta } \\
\text { variant }\end{array}$ & $A D H I B$ & $\mathrm{~T}>\mathrm{C}: \mathrm{CCC}$ & His-Arg & Human \\
\hline CM092574 & Chr4 & $1237564 \mid 3$ & - & DFP & $\begin{array}{l}\text { Asthma, atopic, } \\
\text { association with }\end{array}$ & IL2I & $\mathrm{G}>\mathrm{A}: \mathrm{AAA}$ & Cys-Cys & Human \\
\hline CM03I390 & Chr4 & $14|7085| 8$ & - & DP & $\begin{array}{l}\text { Waist-to-hip ratio, } \\
\text { association with }\end{array}$ & UCPI & C > T:TTT & Ala-Thr & Human \\
\hline CM004732 & Chr5 & $14644 \mid 2$ & - & DP & $\begin{array}{l}\text { Parkinson's disease, } \\
\text { protection against, } \\
\text { association with? }\end{array}$ & SLC6A3 & $\mathrm{T}>\mathrm{C}: \mathrm{CTC}$ & Ser-Ser & $\begin{array}{l}\text { Neanderthal } \\
\text { and } \\
\text { chimpanzee }\end{array}$ \\
\hline CM094298 & Chr5 & 96165006 & - & DFP & $\begin{array}{l}\text { Cervical carcinoma } \\
\text { survival, association } \\
\text { with }\end{array}$ & ERAPI & $\mathrm{C}>\mathrm{G}: \mathrm{GGG}$ & Arg-Pro & Human \\
\hline CM09I0II5 & Chr5 & 131424377 & + & DP & $\begin{array}{l}\text { Graves disease, } \\
\text { association with }\end{array}$ & IL3 & $\mathrm{C}>\mathrm{T}: \mathrm{TTT}$ & Pro-Ser & Human \\
\hline CM043093 & Chr6 & 25958824 & - & DM & $\begin{array}{l}\text { Glycogen storage } \\
\text { disease Ic? }\end{array}$ & SLCI TA3 & $\mathrm{C}>\mathrm{T}: \mathrm{TCC}$ & Gly-Arg & Chimpanzee \\
\hline CM0749II & Chr6 & 39433056 & - & DP & $\begin{array}{l}\text { Coronary heart } \\
\text { disease, association } \\
\text { with }\end{array}$ & KIF6 & $A>G: G G G$ & Trp-Arg & Human \\
\hline CM020385 & Chr6 & 74550153 & + & FP & $\begin{array}{l}\text { Gov platelet antigen } \\
\text { variation }\end{array}$ & $C D 109$ & $\mathrm{~A}>\mathrm{C}: \mathrm{CCC}$ & Tyr-Ser & Human \\
\hline CM993455 & Chr6 & $|322| 406 \mid$ & + & DFP & $\begin{array}{l}\text { Insulin resistance, } \\
\text { association with }\end{array}$ & ENPPI & $\mathrm{A}>\mathrm{C}: \mathrm{CCC}$ & Lys-Gln & Human \\
\hline CM0604I5 & Chr6 & 150156438 & + & FP & $\begin{array}{l}\text { Reduced stability, } \\
\text { association with }\end{array}$ & PCMTI & $A>G: A G G$ & Ile-Val & Ancient \\
\hline CM072043 & Chr6 & 160462998 & + & $\mathrm{FP}$ & $\begin{array}{l}\text { Reduced metformin } \\
\text { uptake, association } \\
\text { with }\end{array}$ & SLC22AI & $\mathrm{C}>\mathrm{T}: \mathrm{TCC}$ & Ser-Phe & Chimpanzee \\
\hline CM005460 & Chr7 & 17345635 & + & $\mathrm{FP}$ & $\begin{array}{l}\text { Higher induced } \\
\text { cytochrome P-450 } \\
\text { (CYP) IAI activity, } \\
\text { association with }\end{array}$ & $A H R$ & $\mathrm{G}>\mathrm{A}: \mathrm{AAA}$ & Arg-Lys & Human \\
\hline CM055287 & Chr7 & 45899194 & + & DP & $\begin{array}{l}\text { Renal function in } \\
\text { diabetes, association } \\
\text { with }\end{array}$ & IGFBPI & $A>G: G G A$ & Ile-Met & $\begin{array}{l}\text { Denisova } \\
\text { and } \\
\text { chimpanzee }\end{array}$ \\
\hline CM0728I4 & Chr7 & 86894112 & - & DM & $\begin{array}{l}\text { Intrahepatic } \\
\text { cholestasis, familial } \\
\text { progressive? }\end{array}$ & $A B C B 4$ & $\mathrm{~T}>\mathrm{C}: \mathrm{CCC}$ & Arg-Gly & Human \\
\hline
\end{tabular}


Table S2. Continued

\begin{tabular}{|c|c|c|c|c|c|c|c|c|c|}
\hline $\begin{array}{l}\text { HGMD } \\
\text { Acc }\end{array}$ & Chr & Location & Strand & Tag & Disease & Gene & Mutation & AA seq & Type \\
\hline CM064968 & Chr7 & 91468556 & + & DP & $\begin{array}{l}\text { Colorectal cancer, } \\
\text { increased risk, } \\
\text { association with }\end{array}$ & AKAP9 & G > T:TTT & Met-Ile & Human \\
\hline CM930596 & Chr7 & 94775382 & - & DFP & $\begin{array}{l}\text { Longevity, association } \\
\text { with }\end{array}$ & PONI & $\mathrm{T}>\mathrm{C}: \mathrm{CCC}$ & Gln-Arg & Human \\
\hline CM050323 & Chr7 & 107129530 & + & DM & Pendred syndrome? & SLC26A4 & $\mathrm{T}>\mathrm{G}: \mathrm{GTG}$ & Val-Gly & $\begin{array}{l}\text { Neanderthal } \\
\text { and } \\
\text { chimpanzee }\end{array}$ \\
\hline CM060083 & Chr7 & 122422409 & - & DP & $\begin{array}{l}\text { Alcohol dependence, } \\
\text { risk, association with }\end{array}$ & TAS2RI 6 & $\mathrm{~A}>\mathrm{C}: \mathrm{CAA}$ & Asn-Lys & Chimpanzee \\
\hline CM03I370 & Chr7 & $14|3| 9073$ & - & DP & $\begin{array}{l}\text { Phenylthiocarbamide } \\
\text { taste sensitivity, } \\
\text { association with }\end{array}$ & TAS2R38 & $\mathrm{T}>\mathrm{C}: \mathrm{CCC}$ & Ile-Val & Human \\
\hline CM03।368 & Chr7 & 141319814 & - & DP & $\begin{array}{l}\text { Phenylthiocarbamide } \\
\text { taste sensitivity, } \\
\text { association with }\end{array}$ & TAS2R38 & $\mathrm{C}>\mathrm{G}: \mathrm{GGG}$ & Ala-Pro & Human \\
\hline CM08I694 & Chr8 & 6466450 & + & DP & $\begin{array}{l}\text { Cranial volume, } \\
\text { association with }\end{array}$ & $\mathrm{MCPHI}$ & $\mathrm{C}>\mathrm{T}: \mathrm{TTT}$ & Ala-Val & Human \\
\hline CM024569 & Chr8 & 18124476 & + & $\mathrm{FP}$ & $\begin{array}{l}\text { Increased enzymatic } \\
\text { activity, association } \\
\text { with }\end{array}$ & NATI & $\mathrm{T}>\mathrm{G}: \mathrm{GTG}$ & Ser-Ala & $\begin{array}{l}\text { Neanderthal } \\
\text { and } \\
\text { chimpanzee }\end{array}$ \\
\hline CM983990 & Chr8 & 22032655 & - & DM & Alopecia universalis? & $H R$ & $\mathrm{~T}>\mathrm{C}: \mathrm{CCC}$ & Thr-Ala & Human \\
\hline CM05743I & Chr8 & 27518398 & - & DP & $\begin{array}{l}\text { Preeclampsia \& } \\
\text { essential } \\
\text { hypertension, } \\
\text { association with? }\end{array}$ & $C L U$ & $A>G: G G G$ & His-His & Human \\
\hline CM9500I7 & Chr8 & 37942955 & - & DFP & $\begin{array}{l}\text { Hyperinsulinaemia, } \\
\text { association with }\end{array}$ & ADRB3 & A $>$ G:GGG & Trp-Arg & Human \\
\hline CM099178 & Chr8 & II 8899878 & - & DM & $\begin{array}{l}\text { Multiple } \\
\text { osteochondromas }\end{array}$ & EXTI & C $>$ T:TCC & Val-Ile & Chimpanzee \\
\hline CM08I76I & Chr8 & |43758933 & + & DFP & $\begin{array}{l}\text { Gastric cancer, } \\
\text { diffuse-type, } \\
\text { association with }\end{array}$ & PSCA & $\mathrm{C}>\mathrm{T}: \mathrm{TTT}$ & Thr-Met & Human \\
\hline CM094855 & Chr9 & |47| 2477 & - & DP & $\begin{array}{l}\text { Low bone mineral } \\
\text { density, association } \\
\text { with }\end{array}$ & CERI & $\mathrm{G}>\mathrm{C}: \mathrm{CGC}$ & Ala-Gly & $\begin{array}{l}\text { Neanderthal } \\
\text { and } \\
\text { chimpanzee }\end{array}$ \\
\hline CM940804 & Chr9 & 34639442 & + & DFP & $\begin{array}{l}\text { Galactosaemia, } \\
\text { Duarte variant }\end{array}$ & GALT & A $>$ G:GAG & Asn-Asp & $\begin{array}{l}\text { Neanderthal } \\
\text { and } \\
\text { chimpanzee }\end{array}$ \\
\hline
\end{tabular}

Continued 
Table S2. Continued

\begin{tabular}{|c|c|c|c|c|c|c|c|c|c|}
\hline $\begin{array}{l}\text { HGMD } \\
\text { Acc }\end{array}$ & Chr & Location & Strand & Tag & Disease & Gene & Mutation & AA seq & Type \\
\hline CM07I685 & Chr9 & 89511843 & + & DP & $\begin{array}{l}\text { Inactivation of } \\
\text { extracellular } \\
\text { signal-regulated } \\
\text { kinase (ERK)-induced } \\
\text { apoptosis, } \\
\text { association with }\end{array}$ & DAPKI & $A>G: A G G$ & Asn-Ser & Ancient \\
\hline CM990005 & Chr9 & 106626574 & - & FP & $\begin{array}{l}\text { Higher plasma HDL } \\
\text { cholesterol, } \\
\text { association with }\end{array}$ & $A B C A I$ & $\mathrm{~T}>\mathrm{C}: \mathrm{CCC}$ & Ile-Met & Human \\
\hline CM0910II4 & ChrX & $774 \mid 4973$ & - & DP & $\begin{array}{l}\text { Asthma, association } \\
\text { with }\end{array}$ & CYSLTRI & $\mathrm{G}>\mathrm{A}: \mathrm{AAA}$ & Phe-Phe & Human \\
\hline CM085353 & ChrX & 149390017 & + & DM & Hypospadias & MAMLDI & $\mathrm{T}>\mathrm{C}: \mathrm{CYC}$ & Val-Ala & Unsure \\
\hline CR043I 64 & Chrl & 43575707 & + & DP & $\begin{array}{l}\text { Platelet count, } \\
\text { association with? }\end{array}$ & $M P L$ & $C>A: A A A$ & & Human \\
\hline CR060579 & Chrl & III 020443 & - & DP & $\begin{array}{l}\text { Low insulin } \\
\text { sensitivity, } \\
\text { association with }\end{array}$ & KCNA3 & $\mathrm{T}>\mathrm{C}: \mathrm{TCC}$ & & Ancient \\
\hline CR05779I & Chrl & 111571946 & + & FP & $\begin{array}{l}\text { Increased promoter } \\
\text { activity, association } \\
\text { with }\end{array}$ & CHI3L2 & $\mathrm{G}>\mathrm{T}: \mathrm{GGT}$ & & Neanderthal \\
\hline CR031479 & Chrl & $|70894| 2 \mid$ & + & DFP & $\begin{array}{l}\text { Systemic lupus } \\
\text { erythematosus (SLE), } \\
\text { association with }\end{array}$ & FASLG & $\mathrm{C}>\mathrm{T}: \mathrm{TTT}$ & & Human \\
\hline CR025943 & Chrl & $2289|702|$ & - & DP & $\begin{array}{l}\text { Increased } \\
\text { angiotensinogen } \\
\text { levels, association } \\
\text { with? }\end{array}$ & $A G T$ & $\mathrm{G}>\mathrm{A}: \mathrm{AGG}$ & & Chimpanzee \\
\hline CRI02882 & ChrlO & 64279946 & - & DFP & SLE, association with & $E G R 2$ & $\mathrm{C}>\mathrm{T}: \mathrm{TCC}$ & & Chimpanzee \\
\hline CRI02883 & ChrlO & 64280724 & - & DFP & SLE, association with & EGR2 & $\mathrm{T}>\mathrm{C}: \mathrm{CTT}$ & & Chimpanzee \\
\hline CR0723I3 & Chrlo & 94452862 & + & DP & $\begin{array}{l}\text { Diabetes, type } 2 \text {, } \\
\text { association with? }\end{array}$ & HHEX & $\mathrm{C}>\mathrm{T}: \mathrm{TCC}$ & & Chimpanzee \\
\hline CR942079 & Chrlo & $104587 \mid 42$ & - & DP & $\begin{array}{l}\text { Polycystic ovaries, } \\
\text { association with }\end{array}$ & CYPI TAI & $A>G: G G G$ & & Human \\
\hline CROI 2509 & Chrll & $344 \mid 6293$ & + & DP & $\begin{array}{l}\text { Hypertension, } \\
\text { susceptibility to, } \\
\text { association with }\end{array}$ & CAT & $G>A: A G A$ & & $\begin{array}{l}\text { Neanderthal } \\
\text { and } \\
\text { chimpanzee }\end{array}$ \\
\hline CR072303 & Chrll & 44212190 & + & DP & $\begin{array}{l}\text { Diabetes, type } 2 \text {, } \\
\text { reduced risk, } \\
\text { association with? }\end{array}$ & EXT2 & $\mathrm{C}>\mathrm{T}: \mathrm{CTT}$ & & Ancient \\
\hline
\end{tabular}


Table S2. Continued

\begin{tabular}{|c|c|c|c|c|c|c|c|c|}
\hline $\begin{array}{l}\text { HGMD } \\
\text { Acc }\end{array}$ & Chr & Location & Strand & Tag & Disease & Gene & Mutation AA seq & Type \\
\hline CR035965 & Chrll & 45863406 & + & DFP & $\begin{array}{l}\text { Alzheimer's disease, } \\
\text { association with }\end{array}$ & MAPK8IPI & $A>G: G G G$ & Human \\
\hline CR094845 & Chrll & 74539529 & + & $\mathrm{FP}$ & $\begin{array}{l}\text { Increased mRNA } \\
\text { expression, } \\
\text { association with }\end{array}$ & SLCO2BI & $G>A: A A A$ & Human \\
\hline CR045957 & Chrll & 102101690 & - & DFP & $\begin{array}{l}\text { Preterm premature } \\
\text { rupture of } \\
\text { membranes, } \\
\text { association with? }\end{array}$ & MMP8 & $\mathrm{G}>\mathrm{A}: \mathrm{GGA}$ & Neanderthal \\
\hline CR0255I0 & Chrll & 102331749 & - & $\mathrm{FP}$ & $\begin{array}{l}\text { Increased } \\
\text { transcriptional } \\
\text { activity, association } \\
\text { with }\end{array}$ & MMPI3 & $\mathrm{C}>\mathrm{T}: \mathrm{TCC}$ & Chimpanzee \\
\hline CR03।478 & Chrl2 & 10203556 & - & DP & $\begin{array}{l}\text { Alzheimer disease, } \\
\text { reduced risk, } \\
\text { association with }\end{array}$ & OLRI & $\mathrm{G}>\mathrm{A}: \mathrm{AAG}$ & $\begin{array}{l}\text { Denisova } \\
\text { and } \\
\text { chimpanzee }\end{array}$ \\
\hline CR08203I & Chrl2 & 55796928 & - & DP & $\begin{array}{l}\text { Schistosomiasis } \\
\text { infection, association } \\
\text { with }\end{array}$ & STAT6 & $\mathrm{C}>\mathrm{T}: \mathrm{TTT}$ & Human \\
\hline CR087739 & Chrl3 & 42046024 & + & DFP & $\begin{array}{l}\text { Bone mineral density } \\
\text { in osteoporosis, } \\
\text { association with? }\end{array}$ & TNFSFII & $\mathrm{C}>\mathrm{T}: \mathrm{CTC}$ & Denisovan \\
\hline CR080758 & Chrl3 & 45577313 & - & $\mathrm{FP}$ & $\begin{array}{l}\text { Increased promoter } \\
\text { activity, association } \\
\text { with }\end{array}$ & CPB2 & $\mathrm{T}>\mathrm{C}: \mathrm{CTT}$ & Chimpanzee \\
\hline CR994765 & Chrl3 & II 2807756 & + & DFP & $\begin{array}{l}\text { Reduced plasma F7 } \\
\text { levels, association } \\
\text { with }\end{array}$ & F7 & $\mathrm{G}>\mathrm{T}: \mathrm{CTT}$ & Unsure \\
\hline CR06666I & Chrl5 & 49336891 & - & DP & $\begin{array}{l}\text { Alzheimer's disease } \\
\text { in apolipoprotein E4 } \\
\text { (APOE4) carriers, } \\
\text { increased risk, } \\
\text { association with }\end{array}$ & CYPI9AI & $\mathrm{G}>\mathrm{A}: \mathrm{AAA}$ & Human \\
\hline CR002I54 & Chrl5 & $565 \mid 1231$ & + & DP & $\begin{array}{l}\text { Dyslipidaemia and } \\
\text { insulin resistance, } \\
\text { association with }\end{array}$ & LIPC & $\mathrm{G}>\mathrm{A}: \mathrm{AGG}$ & Chimpanzee \\
\hline CR993820 & Chrl5 & 72828970 & + & DFP & $\begin{array}{l}\text { Increased activity in } \\
\text { smokers, association } \\
\text { with }\end{array}$ & CYPIA2 & $C>A: A A A$ & Human \\
\hline
\end{tabular}


Table S2. Continued

\begin{tabular}{|c|c|c|c|c|c|c|c|c|c|}
\hline $\begin{array}{l}\text { HGMD } \\
\text { Acc }\end{array}$ & Chr & Location & Strand & Tag & Disease & Gene & Mutation & AA seq & Type \\
\hline CRI02I87 & Chrl6 & 13921167 & + & DFP & $\begin{array}{l}\text { Bladder cancer, } \\
\text { increased risk, } \\
\text { association with }\end{array}$ & ERCC4 & $\mathrm{A}>\mathrm{C}: \mathrm{CAA}$ & & Chimpanzee \\
\hline CR066332 & Chrl6 & 54244319 & + & DFP & $\begin{array}{l}\text { Attention-deficit } \\
\text { hyperactivity } \\
\text { disorder, association } \\
\text { with }\end{array}$ & SLC6A2 & A $>$ T:ATA & & Denisovan \\
\hline CR000229 & Chrl6 & 55552737 & + & DFP & $\begin{array}{l}\text { Higher HDL } \\
\text { cholesterol level, } \\
\text { association with }\end{array}$ & CETP & $C>A: A A A$ & & Human \\
\hline CR0840I2 & Chrl7 & 25549137 & - & $\mathrm{FP}$ & $\begin{array}{l}\text { Increased expression, } \\
\text { association with }\end{array}$ & SLC6A4 & $\mathrm{A}>\mathrm{C}: \mathrm{CCC}$ & & Human \\
\hline CR03588I & Chrl7 & 29706729 & + & FP & $\begin{array}{l}\text { Increased monocyte } \\
\text { chemoattractant } \\
\text { protein-4 (MCP-4) } \\
\text { plasma levels, } \\
\text { association with }\end{array}$ & $\mathrm{CCL} / 3$ & $\mathrm{C}>\mathrm{T}: \mathrm{CCT}$ & & Neanderthal \\
\hline CR003707 & Chrl7 & 31231893 & - & DFP & $\begin{array}{l}\text { Atopic dermatitis, } \\
\text { association with }\end{array}$ & CCL5 & C > T:TTT & & Human \\
\hline CR078280 & Chrl7 & 35323475 & - & DP & $\begin{array}{l}\text { Asthma, increased } \\
\text { risk, association } \\
\text { with? }\end{array}$ & GSDMB & $\mathrm{C}>\mathrm{T}: \mathrm{TTC}$ & & $\begin{array}{l}\text { Denisova } \\
\text { and } \\
\text { chimpanzee }\end{array}$ \\
\hline CR090I98 & Chrl7 & 38531642 & - & $\mathrm{FP}$ & $\begin{array}{l}\text { Promoter activity, } \\
\text { association with }\end{array}$ & BRCAI & $\mathrm{T}>\mathrm{C}: \mathrm{CCC}$ & & Human \\
\hline CR052976 & Chrl7 & 43163827 & + & DP & $\begin{array}{l}\text { Asthma, } \\
\text { aspirin-induced, } \\
\text { association with }\end{array}$ & $T B \times 2 I$ & $\mathrm{~T}>\mathrm{C}: \mathrm{CCC}$ & & Human \\
\hline CR0840I3 & Chrl7 & 43178034 & + & DP & $\begin{array}{l}\text { Genital herpes } \\
\text { simplex virus- } 2 \\
\text { (HSV-2) infection, } \\
\text { association with? }\end{array}$ & $T B \times 2 I$ & $\mathrm{G}>\mathrm{A}: \mathrm{AAA}$ & & Human \\
\hline CR05I 707 & Chrl9 & 7718733 & - & DFP & $\begin{array}{l}\text { Dengue disease, } \\
\text { protection against, } \\
\text { association with }\end{array}$ & CD209 & $A>G: G G G$ & & Human \\
\hline CR095376 & Chrl9 & 40464739 & + & DP & $\begin{array}{l}\text { Increased liver iron } \\
\text { concentration }\end{array}$ & HAMP & $\mathrm{A}>\mathrm{G}: \mathrm{GGA}$ & & $\begin{array}{l}\text { Denisova } \\
\text { and } \\
\text { chimpanzee }\end{array}$ \\
\hline CR050427 & Chrl9 & 46188969 & + & FP & $\begin{array}{l}\text { CYP2B6 expression, } \\
\text { association with }\end{array}$ & CYP2B6 & $\mathrm{T}>\mathrm{C}: \mathrm{CCC}$ & & Human \\
\hline
\end{tabular}


Table S2. Continued

\begin{tabular}{|c|c|c|c|c|c|c|c|c|}
\hline $\begin{array}{l}\text { HGMD } \\
\text { Acc }\end{array}$ & Chr & Location & Strand & Tag & Disease & Gene & Mutation & Type \\
\hline CR05I 274 & Chrl9 & $54 \mid 49750$ & + & DFP & $\begin{array}{l}\text { Disease progression, } \\
\text { chronic lymphocytic } \\
\text { leukaemia, } \\
\text { association with }\end{array}$ & $B A X$ & $\mathrm{G}>\mathrm{A}: \mathrm{GAG}$ & Denisovan \\
\hline CROI0588 & Chrl9 & 60077416 & + & DP & $\begin{array}{l}\text { Immunoglobulin A } \\
\text { nephropathy, } \\
\text { association with }\end{array}$ & FCAR & $\mathrm{T}>\mathrm{C}: \mathrm{CCC}$ & Human \\
\hline CR05I 277 & Chr2 & 69468799 & - & DP & $\begin{array}{l}\text { Obesity, association } \\
\text { with }\end{array}$ & GFPTI & $\mathrm{C}>\mathrm{T}: \mathrm{TTT}$ & Human \\
\hline CR025220 & Chr2 & 234330398 & + & DFP & $\begin{array}{l}\text { Hyperbilirubinaemia, } \\
\text { association with }\end{array}$ & UGTIAI & $\mathrm{T}>\mathrm{G}: \mathrm{GGG}$ & Human \\
\hline CR075263 & Chr20 & 17370063 & + & DP & $\begin{array}{l}\text { Diabetes, type } 2 \text {, } \\
\text { association with }\end{array}$ & PCSK2 & $\mathrm{T}>\mathrm{C}: \mathrm{CCC}$ & Human \\
\hline CR077665 & Chr20 & 44066518 & + & $\mathrm{FP}$ & $\begin{array}{l}\text { Increased expression, } \\
\text { association with? }\end{array}$ & MMP9 & C > T:TTT & Human \\
\hline CR078I66 & Chr2I & 33619134 & + & FP & $\begin{array}{l}\text { Increased expression, } \\
\text { association with }\end{array}$ & IFNARI & $\mathrm{T}>\mathrm{C}: \mathrm{CCT}$ & $\begin{array}{l}\text { Denisova } \\
\text { and } \\
\text { chimpanzee }\end{array}$ \\
\hline CR054260 & Chr2I & 38590628 & + & $\mathrm{FP}$ & $\begin{array}{l}\text { Promoter activity, } \\
\text { association with }\end{array}$ & KCNJI5 & T > G:GTT & Chimpanzee \\
\hline CR096274 & Chr2I & 42492734 & + & DFP & $\begin{array}{l}\text { Coronary artery } \\
\text { disease, severity, } \\
\text { association with }\end{array}$ & $A B C G I$ & $\mathrm{~T}>\mathrm{G}: \mathrm{GGT}$ & $\begin{array}{l}\text { Denisova } \\
\text { and } \\
\text { chimpanzee }\end{array}$ \\
\hline CR032439 & Chr3 & 12328198 & + & DFP & $\begin{array}{l}\text { Increased height/lipid } \\
\text { metabolism, } \\
\text { association with }\end{array}$ & PPARG & $\mathrm{C}>\mathrm{G}: \mathrm{GGG}$ & Human \\
\hline CR066664 & Chr3 & 129680794 & - & DP & $\begin{array}{l}\text { Coronary artery } \\
\text { disease, association } \\
\text { with }\end{array}$ & GATA2 & $\mathrm{G}>\mathrm{A}: \mathrm{AGG}$ & Chimpanzee \\
\hline CROI4438 & Chr3 & 185572960 & - & DP & $\begin{array}{l}\text { Myocardial infarction, } \\
\text { association with }\end{array}$ & THPO & C > T:TTT & Human \\
\hline CR004797 & Chr4 & 26101320 & - & DP & $\begin{array}{l}\text { Higher percentage } \\
\text { body fat, association } \\
\text { with }\end{array}$ & CCKAR & $C>A: A C C$ & Chimpanzee \\
\hline CR045948 & Chr4 & 69995928 & + & $\mathrm{FP}$ & $\begin{array}{l}\text { Promoter activity, } \\
\text { association with }\end{array}$ & UGT2B7 & $\mathrm{G}>\mathrm{A}: \mathrm{AAA}$ & Human \\
\hline CR025435 & Chr4 & 111053559 & + & DFP & $\begin{array}{l}\text { Malignant melanoma, } \\
\text { association with }\end{array}$ & EGF & $\mathrm{A}>\mathrm{G}: \mathrm{GGG}$ & Human \\
\hline
\end{tabular}


Table S2. Continued

\begin{tabular}{|c|c|c|c|c|c|c|c|c|c|}
\hline $\begin{array}{l}\text { HGMD } \\
\text { Acc }\end{array}$ & Chr & Location & Strand & Tag & Disease & Gene & Mutation & AA seq & Type \\
\hline CR057903 & Chr4 & I55703465 & + & DFP & $\begin{array}{l}\text { Cerebral infarction, } \\
\text { association with }\end{array}$ & $F G B$ & $\mathrm{C}>\mathrm{T}: \mathrm{TTT}$ & & Human \\
\hline CR07I28I & Chr4 & I56348632 & + & DP & $\begin{array}{l}\text { Obesity, association } \\
\text { with }\end{array}$ & NPY2R & $\mathrm{C}>\mathrm{T}: \mathrm{TTT}$ & & Human \\
\hline CR07I 289 & Chr5 & 1499389 & - & DP & $\begin{array}{l}\text { Attention-deficit } \\
\text { hyperactivity } \\
\text { disorder, association } \\
\text { with }\end{array}$ & SLC6A3 & $\mathrm{A}>\mathrm{G}: \mathrm{GGG}$ & & Human \\
\hline CR086597 & Chr5 & II043464| & + & FP & $\begin{array}{l}\text { Increased promoter } \\
\text { activity, association } \\
\text { with }\end{array}$ & TSLP & $\mathrm{C}>\mathrm{T}: \mathrm{TCC}$ & & Chimpanzee \\
\hline CR0355I3 & Chr5 & $|3| 43674 \mid$ & + & DP & $\begin{array}{l}\text { Reduced severity in } \\
\text { atopic dermatitis, } \\
\text { association with }\end{array}$ & CSF2 & $A>C: C C C$ & & Human \\
\hline CROI5845 & Chr5 & 132020708 & + & DP & $\begin{array}{l}\text { Asthma, association } \\
\text { with }\end{array}$ & ILI3 & C > T:TTC & & $\begin{array}{l}\text { Denisova } \\
\text { and } \\
\text { chimpanzee }\end{array}$ \\
\hline CR0820I8 & Chr6 & 78227843 & - & DFP & $\begin{array}{l}\text { Aggressive behaviour, } \\
\text { association with }\end{array}$ & $H T R I B$ & $\mathrm{C}>\mathrm{T}: \mathrm{TCC}$ & & Chimpanzee \\
\hline CR073540 & Chr6 & 131935252 & + & DP & $\begin{array}{l}\text { Myocardial infarction, } \\
\text { association with }\end{array}$ & ARGI & $\mathrm{G}>\mathrm{T}: \mathrm{TTT}$ & & Human \\
\hline CR052970 & Chr6 & 132254387 & + & DP & $\begin{array}{l}\text { Obesity, association } \\
\text { with }\end{array}$ & ENPPI & $A>G: G G G$ & & Human \\
\hline CR075243 & Chr6 & $|323| 4950$ & - & DFP & $\begin{array}{l}\text { Systemic sclerosis, } \\
\text { association with }\end{array}$ & CTGF & $\mathrm{C}>\mathrm{G}: \mathrm{CGG}$ & & Ancient \\
\hline CR075274 & Chr6 & $1330770 \mid 8$ & - & DP & $\begin{array}{l}\text { HDL cholesterol } \\
\text { concentration, } \\
\text { association with }\end{array}$ & VNNI & $A>C: C C C$ & & Human \\
\hline CR077383 & Chr6 & $15440 \mid 054$ & + & FP & $\begin{array}{l}\text { Increased promoter } \\
\text { activity, association } \\
\text { with }\end{array}$ & OPRMI & $A>G: G G G$ & & Human \\
\hline CR066667 & Chr7 & 30969948 & + & DP & $\begin{array}{l}\text { Breast cancer, } \\
\text { decreased risk, } \\
\text { association with }\end{array}$ & GHRHR & $\mathrm{C}>\mathrm{T}: \mathrm{TTT}$ & & Human \\
\hline CR092300 & Chr7 & III902894 & + & DFP & $\begin{array}{l}\text { Severity in cystic } \\
\text { fibrosis, association } \\
\text { with }\end{array}$ & IFRDI & $\mathrm{C}>\mathrm{T}: \mathrm{TTT}$ & & Human \\
\hline CR068449 & Chr7 & $|2838| 96 \mid$ & + & DP & $\begin{array}{l}\text { SLE, association } \\
\text { with? }\end{array}$ & IRF5 & $\mathrm{C}>\mathrm{T}: \mathrm{TTT}$ & & Human \\
\hline
\end{tabular}


Table S2. Continued

\begin{tabular}{|c|c|c|c|c|c|c|c|c|}
\hline $\begin{array}{l}\text { HGMD } \\
\text { Acc }\end{array}$ & Chr & Location & Strand & Tag & Disease & Gene & AA seq & Type \\
\hline CR022507 & Chr7 & $13635 \mid 848$ & + & DP & $\begin{array}{l}\text { Major depression in } \\
\text { women, association } \\
\text { with }\end{array}$ & CHRM2 & $\mathrm{T}>\mathrm{A}: \mathrm{AAA}$ & Human \\
\hline CR97I950 & Chr8 & |984095| & + & $\mathrm{FP}$ & $\begin{array}{l}\text { Lower plasma } \\
\text { triglyceride level, } \\
\text { association with }\end{array}$ & $L P L$ & $\mathrm{~T}>\mathrm{G}: \mathrm{GGG}$ & Human \\
\hline CR023703 & Chr8 & 120034205 & - & DP & $\begin{array}{l}\text { Decreased bone } \\
\text { mineral density, } \\
\text { association with? }\end{array}$ & TNFRSFI I B & $\mathrm{C}>\mathrm{T}: \mathrm{TTT}$ & Human \\
\hline CR08400I & Chr9 & 70877744 & + & DP & $\begin{array}{l}\text { Myocardial infarction, } \\
\text { association with }\end{array}$ & $F X N$ & C $>$ T:TTT & Human \\
\hline CRI02I76 & Chr9 & 100952292 & + & DFP & $\begin{array}{l}\text { Breast cancer, } \\
\text { association with }\end{array}$ & TGFBRI & $A>G: G G G$ & Human \\
\hline CR020827 & Chr9 & |0673027| & - & DP & $\begin{array}{l}\text { Increased risk of } \\
\text { coronary artery } \\
\text { disease, association } \\
\text { with }\end{array}$ & $A B C A I$ & $\mathrm{G}>\mathrm{A}: \mathrm{AAA}$ & Human \\
\hline CR045560 & Chr9 & 106730659 & - & FP & $\begin{array}{l}\text { Reduced plasma } \\
\text { HDL cholesterol, } \\
\text { association with }\end{array}$ & $A B C A I$ & C $>$ G:GGG & Human \\
\hline CR091269 & Chr9 & II 6608587 & - & DFP & $\begin{array}{l}\text { Crohn's disease, } \\
\text { susceptibility to, } \\
\text { association with }\end{array}$ & TNFSFI 5 & $A>G: G G G$ & Human \\
\hline CR034594 & Chr9 & 124172343 & + & $\mathrm{FP}$ & $\begin{array}{l}\text { Inhibition of } \\
\text { prostaglandin } \mathrm{H} 2 \\
\text { formation, } \\
\text { association with? }\end{array}$ & PTGSI & $A>G: G A G$ & $\begin{array}{l}\text { Neanderthal } \\
\text { and } \\
\text { chimpanzee }\end{array}$ \\
\hline CR054255 & Chr9 & 127043845 & - & DP & $\begin{array}{l}\text { Bipolar disorder, } \\
\text { association with? }\end{array}$ & HSPA5 & $\mathrm{T}>\mathrm{C}: \mathrm{CCC}$ & Human \\
\hline CR07738I & ChrX & II 3724838 & + & $\mathrm{FP}$ & $\begin{array}{l}\text { Reduced promoter } \\
\text { activity, association } \\
\text { with }\end{array}$ & HTR2C & $\mathrm{G}>\mathrm{C}: \mathrm{CGG}$ & Chimpanzee \\
\hline CR063398 & ChrX & $1355546 \mid 6$ & + & FP & $\begin{array}{l}\text { Increased soluble } \\
\text { CD40 ligand } \\
\text { (CD40L) levels, } \\
\text { association with }\end{array}$ & CD40LG & A $>$ G:GGG & Human \\
\hline
\end{tabular}


Table S3. PCMs covered by the Denisovan sequence but not the Neanderthal sequence

\begin{tabular}{|c|c|c|c|c|c|c|c|c|c|}
\hline Acc & Chr & Location & Strand & Tag & Disease & Gene & Mutation & AA seq & Type \\
\hline CM0624I9 & chrl & 19483828 & - & DP & $\begin{array}{l}\text { Leukaemia, risk, } \\
\text { association with }\end{array}$ & AKR7A3 & $\mathrm{C}>\mathrm{T}: \mathrm{CT}$ & Asp-Asn & Denisovan \\
\hline CM098300 & chrl & 24074507 & - & DFP & $\begin{array}{l}\text { Eating disorders, } \\
\text { association with }\end{array}$ & CNR2 & $\mathrm{T}>\mathrm{C}: \mathrm{CC}$ & Gln-Arg & Ancestral \\
\hline CM066774 & chrl & 110267989 & + & DP & $\begin{array}{l}\text { Periodontitis, } \\
\text { association with? }\end{array}$ & CSFI & $\mathrm{T}>\mathrm{C}: \mathrm{CC}$ & Leu-Pro & Ancestral \\
\hline CM094244 & chrl & 111656412 & + & $\mathrm{FP}$ & $\begin{array}{l}\text { Increased enzyme } \\
\text { activity, association } \\
\text { with? }\end{array}$ & CHIA & $A>G: G A$ & Asn-Asp & Derived \\
\hline CM094243 & chrl & III65646| & + & DFP & $\begin{array}{l}\text { Asthma, protection } \\
\text { against, association } \\
\text { with? }\end{array}$ & $\mathrm{CHIA}$ & $\mathrm{G}>\mathrm{T}: \mathrm{TG}$ & Arg-Met & Derived \\
\hline CM084968 & chrl & I 50552554 & - & DP & $\begin{array}{l}\text { Psoriasis, increased } \\
\text { risk, association with }\end{array}$ & FLG & $G>A: A G$ & Pro-Ser & Derived \\
\hline CM067657 & chrl & |5659|049 & + & DP & $\begin{array}{l}\text { Guillain-Barré } \\
\text { syndrome, reduced } \\
\text { risk, association with }\end{array}$ & $C D / E$ & $A>G: G G$ & Gln-Arg & Ancestral \\
\hline CM033904 & chrl & $1694447 \mid 4$ & + & $\mathrm{FP}$ & $\begin{array}{l}\text { Flavin-containing } \\
\text { monooxygenase } 2 \\
\text { (FMO2) gene variant }\end{array}$ & FMO2 & $\mathrm{T}>\mathrm{C}: \mathrm{CC}$ & Term-Gln & Ancestral \\
\hline CM043273 & chrl & | $9567049 \mid$ & + & DM & Retinitis pigmentosa & CRBI & $G>A: A G$ & Gly-Ser & Derived \\
\hline CM024366 & chrl & 224093029 & + & DFP & $\begin{array}{l}\text { Preeclampsia, } \\
\text { association with }\end{array}$ & EPHXI & $A>G: G A$ & His-Arg & Derived \\
\hline CM994344 & chrlo & 115795046 & + & $\mathrm{FP}$ & $\begin{array}{l}\text { Gain of function, } \\
\text { association with }\end{array}$ & $A D R B I$ & $\mathrm{G}>\mathrm{C}: \mathrm{CC}$ & Gly-Arg & Ancestral \\
\hline CM067436 & chrll & 7020956 & + & DM & $\begin{array}{l}\text { Spermatogenic } \\
\text { failure }\end{array}$ & NLRP/ 4 & $\mathrm{G}>\mathrm{A}: \mathrm{AG}$ & Ala-Thr & Derived \\
\hline CM043536 & chrll & 47326617 & - & DM & $\begin{array}{l}\text { Cardiomyopathy, } \\
\text { hypertrophic? }\end{array}$ & MYBPC3 & $\mathrm{T}>\mathrm{C}: \mathrm{CT}$ & Ser-Gly & Derived \\
\hline CM035848 & chrll & 57739196 & + & $\mathrm{FP}$ & $\begin{array}{l}\text { Olfactory receptor } \\
\text { deficiency? }\end{array}$ & ORISI & $\mathrm{G}>\mathrm{A}: \mathrm{GA}$ & Arg-His & Denisovan \\
\hline CM087504 & chrll & 102218830 & - & DP & $\begin{array}{l}\text { Blood pressure, } \\
\text { association with }\end{array}$ & MMP3 & $\mathrm{T}>\mathrm{C}: \mathrm{CC}$ & Lys-Glu & Ancestral \\
\hline CM04I24I & chrll & 112776038 & + & $\mathrm{FP}$ & $\begin{array}{l}\text { Reduced dopamine } \\
\text { D2 receptor } \\
\text { (DRD2) receptor } \\
\text { density, association } \\
\text { with? }\end{array}$ & ANKKI & $G>A: A A$ & Glu-Lys & Ancestral \\
\hline
\end{tabular}


Table S3. Continued

\begin{tabular}{|c|c|c|c|c|c|c|c|c|c|}
\hline Acc & Chr & Location & Strand & Tag & Disease & Gene & Mutation & AA seq & Type \\
\hline CM082943 & chrll & II 8720796 & - & DM & $\begin{array}{l}\text { Primary } \\
\text { angle-closure } \\
\text { glaucoma? }\end{array}$ & MFRP & $\mathrm{C}>\mathrm{T}: \mathrm{TT}$ & Arg-His & Ancestral \\
\hline CM0750I8 & chrll & 130255852 & - & DP & $\begin{array}{l}\text { Coronary heart } \\
\text { disease, association } \\
\text { with }\end{array}$ & SNX19 & $A>C: C C$ & Leu-Arg & Ancestral \\
\hline CM091988 & $\mathrm{chr} / 2$ & 32913201 & - & DM & $\begin{array}{l}\text { Arrhythmogenic } \\
\text { right ventricular } \\
\text { cardiomyopathy }\end{array}$ & PKP2 & $A>G: G G$ & Leu-Pro & Ancestral \\
\hline CM0876I8 & $\mathrm{chr} / 2$ & 56152088 & + & DFP & $\begin{array}{l}\text { Inflammatory bowel } \\
\text { disease, association } \\
\text { with }\end{array}$ & GLII & $\mathrm{G}>\mathrm{C}: \mathrm{CC}$ & Glu-Gln & Ancestral \\
\hline CM098354 & $\mathrm{chrl} 2$ & 120099486 & + & FP & $\begin{array}{l}\text { Altered function, } \\
\text { association with }\end{array}$ & $P 2 R \times 7$ & $G>A: A A$ & Ala-Thr & Ancestral \\
\hline CM065I86 & $\operatorname{chr} / 3$ & 38162690 & + & DP & $\begin{array}{l}\text { Colorectal cancer, } \\
\text { increased risk, } \\
\text { association with }\end{array}$ & FREM2 & $\mathrm{T}>\mathrm{C}: \mathrm{CC}$ & Phe-Ser & Ancestral \\
\hline CM063919 & $\operatorname{chrl3}$ & 45546095 & - & FP & $\begin{array}{l}\text { Higher } \\
\text { thrombin-activatable } \\
\text { fibrinolysis inhibitor } \\
\text { (TAFI) antigen levels, } \\
\text { association with }\end{array}$ & CPB2 & $\mathrm{C}>\mathrm{T}: \mathrm{TT}$ & Ala-Thr & Ancestral \\
\hline CM044579 & $\operatorname{chr} / 3$ & 51413355 & - & DM & Wilson disease? & ATP7B & $A>G: G G$ & Val-Ala & Ancestral \\
\hline CM063843 & chrl4 & 19994994 & + & DFP & $\begin{array}{l}\text { Amyotrophic lateral } \\
\text { sclerosis, association } \\
\text { with }\end{array}$ & APEXI & $\mathrm{T}>\mathrm{G}: \mathrm{GG}$ & Asp-Glu & Ancestral \\
\hline CM073244 & chrl4 & 20010446 & + & DP & $\begin{array}{l}\text { Faster cognitive } \\
\text { decline in } \\
\text { Alzheimer's disease, } \\
\text { association with }\end{array}$ & $N P$ & $G>A: A G$ & Gly-Ser & Derived \\
\hline CM068495 & chrl5 & 49316404 & - & DP & $\begin{array}{l}\text { Increased cortical } \\
\text { bone mass density, } \\
\text { association with }\end{array}$ & CYPI9AI & $\mathrm{T}>\mathrm{C}: \mathrm{CC}$ & Val-Val & Ancestral \\
\hline CM045806 & chrl5 & 83248435 & + & FP & $\begin{array}{l}\text { Reduced affinity for } \\
\text { gemcitabine, } \\
\text { association with }\end{array}$ & SLC28AI & $\mathrm{G}>\mathrm{A}: \mathrm{AG}$ & Val-Ile & Derived \\
\hline CMI02885 & chrl6 & 10908349 & + & DP & $\begin{array}{l}\text { Multiple sclerosis, } \\
\text { increased risk, } \\
\text { association with }\end{array}$ & CIITA & $\mathrm{G}>\mathrm{C}: \mathrm{CC}$ & Gly-Ala & Ancestral \\
\hline
\end{tabular}


Table S3. Continued

\begin{tabular}{|c|c|c|c|c|c|c|c|c|c|}
\hline Acc & Chr & Location & Strand & Tag & Disease & Gene & Mutation & AA seq & Type \\
\hline CM093I3I & chrl6 & 55950234 & + & DP & $\begin{array}{l}\text { Helicobacter } \\
\text { pylori-related gastric } \\
\text { carcinoma, } \\
\text { association with }\end{array}$ & CCL22 & $A>C: C C$ & Asp-Ala & Ancestral \\
\hline CM067679 & chrl7 & 7858004 & + & DP & $\begin{array}{l}\text { Lung cancer, } \\
\text { susceptibility to, } \\
\text { association with }\end{array}$ & GUCY2D & $\mathrm{T}>\mathrm{A}: \mathrm{AA}$ & Leu-His & Ancestral \\
\hline CM073339 & chrl7 & 24310977 & - & DM & Febrile seizures? & SEZ6 & $\mathrm{T}>\mathrm{C}: \mathrm{CC}$ & Thr-Ala & Ancestral \\
\hline CM05795I & chrl7 & 37960432 & + & DP & $\begin{array}{l}\text { Endometriosis, } \\
\text { association with }\end{array}$ & $H S D \mid 7 B I$ & $A>G: A G$ & Ser-Gly & Denisovan \\
\hline CM9942I4 & $\operatorname{chrl7}$ & 39808591 & - & DP & $\begin{array}{l}\text { Reduced post-stroke } \\
\text { mortality, association } \\
\text { with }\end{array}$ & ITGA2B & $A>C: G C$ & Ile-Ser & Unsure \\
\hline CM09I892 & chrl7 & 42363569 & + & DP & $\begin{array}{l}\text { Hypertension, } \\
\text { association with }\end{array}$ & GOSR2 & $G>A: A G$ & Arg-Lys & Derived \\
\hline CM091876 & $\operatorname{chrl7}$ & 73642170 & + & DP & $\begin{array}{l}\text { Epidermodysplasia } \\
\text { verruciformis, } \\
\text { susceptibility in HIV, } \\
\text { association with }\end{array}$ & TMC8 & $\mathrm{A}>\mathrm{T}: \mathrm{TA}$ & Asn-lle & Derived \\
\hline CM00083I & chrl9 & 3546794 & - & DP & $\begin{array}{l}\text { Bronchial asthma, } \\
\text { association with }\end{array}$ & $T B X A 2 R$ & $A>G: G G$ & Tyr-Tyr & Ancestral \\
\hline CM030470 & chrl9 & $|804| 45 \mid$ & - & DP & $\begin{array}{l}\text { Tuberculosis, } \\
\text { susceptibility to, } \\
\text { association with }\end{array}$ & $|L| 2 R B \mid$ & $A>G: G G$ & Met-Thr & Ancestral \\
\hline CM044082 & chrl9 & I8407678 & - & DP & $\begin{array}{l}\text { Spina bifida, reduced } \\
\text { risk, association with }\end{array}$ & isynal & $\mathrm{T}>\mathrm{C}: \mathrm{CC}$ & Leu-Leu & Ancestral \\
\hline CM057586 & chrl9 & 40534926 & + & DP & $\begin{array}{l}\text { Increased beta-cell } \\
\text { function, association } \\
\text { with }\end{array}$ & FFARI & $G>A: A A$ & Arg-His & Ancestral \\
\hline CM057545 & chrl9 & 50560149 & - & DP & $\begin{array}{l}\text { Lung adenocarcinoma, } \\
\text { increased risk, } \\
\text { association with }\end{array}$ & ERCC2 & $\mathrm{G}>\mathrm{T}: \mathrm{GT}$ & Arg-Arg & Denisovan \\
\hline CM044227 & chrl9 & 60088712 & + & DP & $\begin{array}{l}\text { Aggressive } \\
\text { periodontitis, } \\
\text { reduced risk, assoc } \\
\text { with }\end{array}$ & FCAR & $A>G: G G$ & Arg-Arg & Ancestral \\
\hline CM003809 & chr2 & $38|5568|$ & - & DP & $\begin{array}{l}\text { Breast or lung } \\
\text { cancer, association } \\
\text { with }\end{array}$ & CYPIBI & $C>A: A A$ & Ala-Ser & Ancestral \\
\hline
\end{tabular}


Table S3. Continued

\begin{tabular}{|c|c|c|c|c|c|c|c|c|c|}
\hline Acc & Chr & Location & Strand & Tag & Disease & Gene & Mutation & AA seq & Type \\
\hline CMI0I950 & chr2 & 98363138 & + & DM & $\begin{array}{l}\text { Progressive cone } \\
\text { dystrophy? }\end{array}$ & CNGA3 & $\mathrm{C}>\mathrm{T}: \mathrm{TC}$ & Pro-Leu & Derived \\
\hline CM092797 & chr2 & 169550992 & - & FP & $\begin{array}{l}\text { Alternate splicing, } \\
\text { association with }\end{array}$ & $A B C B / I$ & $\mathrm{~T}>\mathrm{C}: \mathrm{CT}$ & Gly-Gly & Derived \\
\hline CM066575 & chr2 & 218738088 & - & $\mathrm{DP}$ & $\begin{array}{l}\text { AIDS progression, } \\
\text { protection, } \\
\text { association with }\end{array}$ & IL8RA & $A>C: C C$ & Met-Arg & Ancestral \\
\hline CM057769 & chr2 & 234266408 & + & FP & $\begin{array}{l}\text { Altered enzyme } \\
\text { activity, association } \\
\text { with }\end{array}$ & UGTIAG & $\mathrm{T}>\mathrm{G}: \mathrm{GG}$ & Ser-Ala & Ancestral \\
\hline CM9100I8 & chr2 & 241466189 & + & DP & $\begin{array}{l}\text { Hyperoxaluria, } \\
\text { association with }\end{array}$ & $A G X T$ & $A>G: G G$ & Ile-Met & Ancestral \\
\hline CM053304 & chr20 & 54257212 & + & DP & $\begin{array}{l}\text { Obesity, association } \\
\text { with }\end{array}$ & $M C 3 R$ & $C>A: A A$ & Thr-Lys & Ancestral \\
\hline CM97039I & chr22 & $1833 \mid 207$ & + & DFP & $\begin{array}{l}\text { Schizoaffective } \\
\text { disorder, association } \\
\text { with }\end{array}$ & COMT & $C>\mathrm{G}: \mathrm{GG}$ & Leu-Leu & Ancestral \\
\hline CM961335 & chr22 & 30817700 & + & DM & $\begin{array}{l}\text { Glucose/galactose } \\
\text { malabsorption }\end{array}$ & SLC5AI & $\mathrm{G}>\mathrm{A}: \mathrm{AA}$ & Ala-Thr & Ancestral \\
\hline CM930I87 & chr22 & 40853887 & - & DP & $\begin{array}{l}\text { Parkinson's disease, } \\
\text { association with }\end{array}$ & CYP2D6 & $\mathrm{G}>\mathrm{A}: \mathrm{GA}$ & Arg-Cys & Denisovan \\
\hline CM099899 & chr22 & 41888870 & + & FP & $\begin{array}{l}\text { Increased } \\
\text { pregnenolone levels, } \\
\text { association with }\end{array}$ & TSPO & $A>G: G G$ & Thr-Ala & Ancestral \\
\hline CM025430 & chr4 & $297584 I$ & + & FP & $\begin{array}{l}\text { Activity, association } \\
\text { with }\end{array}$ & GRK4 & $\mathrm{C}>\mathrm{T}: \mathrm{TT}$ & Ala-Val & Ancestral \\
\hline CMOI3959 & $\mathrm{chr} 4$ & 23424760 & - & $\mathrm{DP}$ & $\begin{array}{l}\text { Diabetes, type } 2 \text {, } \\
\text { association with }\end{array}$ & PPARGCIA & $\mathrm{C}>\mathrm{T}: \mathrm{TC}$ & Gly-Ser & Derived \\
\hline CM033593 & chr4 & 100479812 & - & $\mathrm{DP}$ & $\begin{array}{l}\text { Alcoholism, } \\
\text { increased risk, } \\
\text { association with? }\end{array}$ & $A D H I C$ & $\mathrm{~T}>\mathrm{C}: \mathrm{CC}$ & Ile-Val & Ancestral \\
\hline CM064956 & chr4 & 109893565 & - & DP & $\begin{array}{l}\text { Colorectal cancer, } \\
\text { increased risk, } \\
\text { association with }\end{array}$ & $A G X T 2 L I$ & $A>G: G G$ & Ser-Pro & Ancestral \\
\hline CM030066 & $\mathrm{chr} 4$ & 149576925 & - & FP & $\begin{array}{l}\text { Reduced expression, } \\
\text { association with }\end{array}$ & $N R 3 C 2$ & $\mathrm{~T}>\mathrm{C}: \mathrm{TC}$ & Ile-Val & Denisovan \\
\hline CM080365 & $\mathrm{chr} 4$ & $|557| \mid 209$ & + & DP & $\begin{array}{l}\text { Increased clot } \\
\text { stiffness, association } \\
\text { with }\end{array}$ & $F G B$ & $\mathrm{G}>\mathrm{A}: \mathrm{AA}$ & Arg-Lys & Ancestral \\
\hline
\end{tabular}


Table S3. Continued

\begin{tabular}{|c|c|c|c|c|c|c|c|c|c|}
\hline Acc & Chr & Location & Strand & Tag & Disease & Gene & Mutation & AA seq & Type \\
\hline CM057405 & chr4 & $156355 \mid 26$ & + & DP & $\begin{array}{l}\text { Severe obesity, in } \\
\text { men, association } \\
\text { with }\end{array}$ & $N P Y 2 R$ & $\mathrm{C}>\mathrm{T}: \mathrm{TT}$ & Ile-Ile & Ancestral \\
\hline CM067358 & chr5 & $221|434|$ & - & DP & $\begin{array}{l}\text { Lung cancer, } \\
\text { susceptibility to, } \\
\text { association with }\end{array}$ & $\mathrm{CDH} / 2$ & $\mathrm{C}>\mathrm{T}: \mathrm{TT}$ & Val-Met & Ancestral \\
\hline CM094788 & chr5 & $12|44| 107$ & - & DFP & $\begin{array}{l}\text { Breast cancer, } \\
\text { increased risk, in } \\
\text { African American } \\
\text { women, association } \\
\text { with }\end{array}$ & LOX & $\mathrm{C}>\mathrm{T}: \mathrm{TT}$ & Arg-Gln & Ancestral \\
\hline CMOI38I5 & chr5 & |4746| | 48 & + & DP & $\begin{array}{l}\text { Atopy, maternally } \\
\text { inherited, } \\
\text { association with }\end{array}$ & SPINK5 & $\mathrm{G}>\mathrm{A}: \mathrm{GA}$ & Glu-Lys & Denisovan \\
\hline CM083577 & chr6 & 24611569 & + & DFP & $\begin{array}{l}\text { Impaired cognitive } \\
\text { function, association } \\
\text { with }\end{array}$ & ALDH5AI & $\mathrm{C}>\mathrm{T}: \mathrm{TT}$ & His-Tyr & Ancestral \\
\hline CM086|46 & chr6 & 25921129 & - & DP & $\begin{array}{l}\text { Uric acid } \\
\text { concentration, } \\
\text { association with }\end{array}$ & SLCI TAI & $\mathrm{G}>\mathrm{A}: \mathrm{AA}$ & Thr-Ile & Ancestral \\
\hline CM052232 & chr6 & 80683094 & - & DP & $\begin{array}{l}\text { Age-related } \\
\text { maculopathy, } \\
\text { association with }\end{array}$ & ELOVL4 & $\mathrm{T}>\mathrm{C}: \mathrm{CT}$ & Met-Val & Derived \\
\hline CM073245 & chr7 & 34784638 & + & DP & $\begin{array}{l}\text { Panic disorder, in } \\
\text { males, association } \\
\text { with }\end{array}$ & NPSRI & $\mathrm{A}>\mathrm{T}: \mathrm{TT}$ & Asn-lle & Ancestral \\
\hline CM084696 & chr7 & 87017537 & - & DFP & $\begin{array}{l}\text { Parkinson's disease, } \\
\text { association with }\end{array}$ & $A B C B I$ & $A>G: G G$ & Gly-Gly & Ancestral \\
\hline CM09I200 & chr7 & I 29737976 & + & DP & $\begin{array}{l}\text { Prostate cancer, } \\
\text { aggressive } \\
\text { early-onset, } \\
\text { association with }\end{array}$ & CPA4 & $\mathrm{G}>\mathrm{T}: \mathrm{TT}$ & Gly-Cys & Ancestral \\
\hline CM952203 & chr7 & 142350235 & - & $\mathrm{FP}$ & $\begin{array}{l}\text { Kell blood group } \\
\text { variation }\end{array}$ & $K E L$ & $A>G: G A$ & Leu-Pro & Derived \\
\hline CM073993 & chr7 & $150 \mid 88598$ & + & $\mathrm{FP}$ & $\begin{array}{l}\text { Reduced activity, } \\
\text { association with }\end{array}$ & $A B P I$ & $C>G: G G$ & His-Asp & Ancestral \\
\hline CM973386 & chr8 & $|8| 2428 \mid$ & + & $\mathrm{FP}$ & $\begin{array}{l}\text { Increased activity, } \\
\text { association with }\end{array}$ & NATI & $G>A: A G$ & Val-Ile & Derived \\
\hline CM099895 & chr8 & $244 \mid 2708$ & + & DP & $\begin{array}{l}\text { Schizophrenia, } \\
\text { association with }\end{array}$ & ADAM7 & $A>C: C C$ & Asn-His & Ancestral \\
\hline
\end{tabular}


Table S3. Continued

\begin{tabular}{|c|c|c|c|c|c|c|c|c|c|}
\hline Acc & Chr & Location & Strand & Tag & Disease & Gene & Mutation & AA seq & Type \\
\hline CM064954 & chr8 & 26683945 & - & DP & $\begin{array}{l}\text { Hypertension, } \\
\text { association with? }\end{array}$ & $A D R A I A$ & $A>G: G G$ & Cys-Arg & Ancestral \\
\hline CM033767 & chr8 & $274 \mid 4422$ & + & DFP & $\begin{array}{l}\text { Coronary heart } \\
\text { disease, in } \\
\text { Caucasians, } \\
\text { association with }\end{array}$ & $E P H X 2$ & $A>G: G A$ & Lys-Arg & Derived \\
\hline CM034886 & chr8 & 91059655 & - & DP & $\begin{array}{l}\text { Lung cancer, } \\
\text { association with? }\end{array}$ & NBN & $\mathrm{C}>\mathrm{G}: \mathrm{GG}$ & Glu-Gln & Ancestral \\
\hline CM045665 & chr8 & 120033233 & - & DP & $\begin{array}{l}\text { Osteoporotic } \\
\text { fractures, association } \\
\text { with }\end{array}$ & TNFRSFI I B & $\mathrm{G}>\mathrm{C}: \mathrm{CG}$ & Asn-Lys & Derived \\
\hline CM093465 & chr9 & 2181309 & + & DFP & $\begin{array}{l}\text { Schizophrenia, } \\
\text { association with }\end{array}$ & SMARCA2 & $\mathrm{C}>\mathrm{G}: \mathrm{GC}$ & Asp-Glu & Derived \\
\hline CM073190 & chrX & 43475980 & + & DP & $\begin{array}{l}\text { Bipolar disorder, } \\
\text { association with? }\end{array}$ & MAOA & $\mathrm{T}>\mathrm{G}: \mathrm{TG}$ & Arg-Arg & Denisovan \\
\hline CR07232I & chrl & $1184 \mid 858$ & - & DFP & $\begin{array}{l}\text { Diabetes, type, } 2 \text {, } \\
\text { reduced risk, } \\
\text { association with }\end{array}$ & NPPB & $A>G: G G$ & & Ancestral \\
\hline CR080762 & chrl & I 5645754 & + & DM & Pancreatitis, chronic? & CTRC & $\mathrm{T}>\mathrm{C}: \mathrm{CC}$ & & Ancestral \\
\hline CR08076I & chrl & 15645757 & + & DM & Pancreatitis, chronic? & CTRC & $A>G: G G$ & & Ancestral \\
\hline CROI6I87 & chrl & 87101113 & - & FP & $\begin{array}{l}\text { Increased } \\
\text { selenocysteine } \\
\text { insertion sequence } \\
\text { (SECIS) efficiency, } \\
\text { association with }\end{array}$ & $\operatorname{sep} / 5$ & $\mathrm{C}>\mathrm{T}: \mathrm{TT}$ & & Ancestral \\
\hline CR092707 & chrl & 201194130 & - & DFP & $\begin{array}{l}\text { Lower insulin } \\
\text { resistance, } \\
\text { association with }\end{array}$ & ADIPORI & $\mathrm{C}>\mathrm{T}: \mathrm{TT}$ & & Ancestral \\
\hline CR034628 & chrlo & 26545502 & + & DP & $\begin{array}{l}\text { Obesity, association } \\
\text { with? }\end{array}$ & GAD2 & $\mathrm{G}>\mathrm{A}: \mathrm{GA}$ & & Denisovan \\
\hline CR061340 & chrll & 35397552 & - & DFP & $\begin{array}{l}\text { Progressing stroke, } \\
\text { increased risk, } \\
\text { association with }\end{array}$ & SLCIA2 & $\mathrm{T}>\mathrm{G}: \mathrm{GG}$ & & Ancestral \\
\hline CR0682I2 & chrll & 59612604 & + & DFP & $\begin{array}{l}\text { Asthma, } \\
\text { aspirin-intolerant }\end{array}$ & MS4A2 & $\mathrm{T}>\mathrm{C}: \mathrm{CC}$ & & Ancestral \\
\hline CR063407 & chrl4 & 50069895 & - & DP & $\begin{array}{l}\text { Diabetes, type 2, } \\
\text { reduced risk, } \\
\text { association with }\end{array}$ & MAP4K5 & $G>A: A A$ & & Ancestral \\
\hline CR077666 & chrl5 & 71712835 & - & DFP & $\begin{array}{l}\text { Schizophrenia, } \\
\text { reduced risk, } \\
\text { association with? }\end{array}$ & NPTN & $C>A: C A$ & & Denisovan \\
\hline
\end{tabular}


Table S3. Continued

\begin{tabular}{|c|c|c|c|c|c|c|c|c|c|}
\hline Acc & Chr & Location & Strand & Tag & Disease & Gene & Mutation & AA seq & Type \\
\hline CR084880 & chrl7 & 35697157 & + & DFP & $\begin{array}{l}\text { Hepatocellular } \\
\text { carcinoma, reduced } \\
\text { risk, association with }\end{array}$ & $C D C 6$ & $A>G: G G$ & & Ancestral \\
\hline CR087465 & chrl7 & 39785770 & + & DFP & $\begin{array}{l}\text { Frontotemporal } \\
\text { dementia, } \\
\text { association with }\end{array}$ & GRN & $\mathrm{C}>\mathrm{T}: \mathrm{TT}$ & & Ancestral \\
\hline CR035036 & chrl8 & 647685 & + & $\mathrm{FP}$ & $\begin{array}{l}\text { Transcriptional } \\
\text { activity, association } \\
\text { with }\end{array}$ & TYMS & $\mathrm{G}>\mathrm{C}: \mathrm{CC}$ & & Ancestral \\
\hline CR032436 & chrl8 & 45342041 & + & DP & $\begin{array}{l}\text { High-density } \\
\text { lipoprotein (HDL) } \\
\text { cholesterol levels, } \\
\text { association with? }\end{array}$ & LIPG & $A>C: C A$ & & Derived \\
\hline CR087I82 & chrl9 & 44589133 & + & DFP & $\begin{array}{l}\text { Rheumatoid } \\
\text { arthritis, shorter } \\
\text { duration, association } \\
\text { with }\end{array}$ & ZFP36 & $A>G: G G$ & & Ancestral \\
\hline CR035033 & chrl9 & $46 \mid 88301$ & + & FP & $\begin{array}{l}\text { Cytochrome P-450 } \\
\text { (CYP) 2B6 } \\
\text { expression, } \\
\text { association with? }\end{array}$ & CYP2B6 & $\mathrm{T}>\mathrm{C}: \mathrm{CC}$ & & Ancestral \\
\hline CR068525 & chr2 & 69467665 & - & DFP & $\begin{array}{l}\text { Diabetes, type } 2 \text {, } \\
\text { association with }\end{array}$ & GFPTI & $A>G: G G$ & & Ancestral \\
\hline CR077669 & chr2 & 85748849 & - & $\mathrm{FP}$ & $\begin{array}{l}\text { Increased promoter } \\
\text { activity, association } \\
\text { with }\end{array}$ & SFTPB & $\mathrm{T}>\mathrm{G}: \mathrm{GG}$ & & Ancestral \\
\hline CR093507 & chr2 & 168743982 & - & DFP & $\begin{array}{l}\text { Hypertension, } \\
\text { association with }\end{array}$ & STK39 & $A>G: G G$ & & Ancestral \\
\hline CR093026 & chr2 & 169465787 & + & DFP & $\begin{array}{l}\text { Increased insulin } \\
\text { secretion, } \\
\text { association with }\end{array}$ & G6PC2 & $\mathrm{G}>\mathrm{A}: \mathrm{AA}$ & & Ancestral \\
\hline CR073559 & chr2 & $224 I 74588$ & - & DFP & $\begin{array}{l}\text { Hypertension, } \\
\text { association with }\end{array}$ & SCG2 & $\mathrm{C}>\mathrm{T}: \mathrm{TT}$ & & Ancestral \\
\hline CR053505 & chr20 & 4653756 & + & DP & $\begin{array}{l}\text { Creutzfeldt-Jakob } \\
\text { disease, association } \\
\text { with? }\end{array}$ & PRND & $\mathrm{T}>\mathrm{C}: \mathrm{CC}$ & & Ancestral \\
\hline CROI5272 & chr22 & 40858326 & - & $\mathrm{FP}$ & $\begin{array}{l}\text { Intermediate } \\
\text { metaboliser, } \\
\text { association with? }\end{array}$ & CYP2D6 & $\mathrm{C}>\mathrm{G}: \mathrm{GG}$ & & Ancestral \\
\hline CR055620 & chr4 & 75938792 & - & FP & $\begin{array}{l}\text { Promoter activity, } \\
\text { association with }\end{array}$ & BTC & $C>G: G G$ & & Ancestral \\
\hline
\end{tabular}


Table S3. Continued

\begin{tabular}{|c|c|c|c|c|c|c|c|c|c|}
\hline Acc & Chr & Location & Strand & Tag & Disease & Gene & Mutation & AA seq & Type \\
\hline CR093469 & chr6 & 2945302 & + & DFP & $\begin{array}{l}\text { Breast cancer, } \\
\text { decreased risk, } \\
\text { association with }\end{array}$ & NQO2 & $\mathrm{A}>\mathrm{C}: \mathrm{CA}$ & & Derived \\
\hline CR035882 & chr6 & 78230101 & - & DFP & $\begin{array}{l}\text { Suicidal ideation, in } \\
\text { major depression, } \\
\text { association with }\end{array}$ & HTRIB & $A>C: C A$ & & Derived \\
\hline CR025333 & chr6 & 137582213 & - & DFP & $\begin{array}{l}\text { Malaria, } \\
\text { susceptibility, } \\
\text { association with }\end{array}$ & IFNGRI & $A>G: G G$ & & Ancestral \\
\hline CR093919 & chr6 & 153121754 & + & DP & $\begin{array}{l}\text { Pulmonary arterial } \\
\text { hypertension, } \\
\text { idiopathic, } \\
\text { association with? }\end{array}$ & VIP & $\mathrm{T}>\mathrm{C}: \mathrm{CC}$ & & Ancestral \\
\hline CROI6I49 & chr7 & 22732746 & + & FP & $\begin{array}{l}\text { Altered } \\
\text { transcriptional } \\
\text { activity, association } \\
\text { with }\end{array}$ & IL6 & $A>G: G G$ & & Ancestral \\
\hline CR053504 & chr7 & 91995822 & - & FP & $\begin{array}{l}\text { Gene expression, } \\
\text { association with }\end{array}$ & PEXI & $A>G: G A$ & & Derived \\
\hline CR04II38 & chr7 & 99192235 & - & DP & $\begin{array}{l}\text { Prostate cancer, low } \\
\text { aggressiveness, } \\
\text { association with }\end{array}$ & CYP3A4 & $G>A: A G$ & & Derived \\
\hline CR0723I 6 & chr7 & 128376663 & + & FP & $\begin{array}{l}\text { Shorter transcript, } \\
\text { association with }\end{array}$ & IRF5 & $G>A: A A$ & & Ancestral \\
\hline CR962526 & chr8 & $4 \mid 774321$ & - & DM & Spherocytosis & ANKI & $A>G: G A$ & & Derived \\
\hline CR0980I3 & chr9 & 22109195 & + & DFP & $\begin{array}{l}\text { Coronary artery } \\
\text { disease, association } \\
\text { with }\end{array}$ & CDKN2BAS & $\mathrm{C}>\mathrm{T}: \mathrm{CT}$ & & Denisovan \\
\hline CR044772 & chr9 & 99499399 & - & DP & $\begin{array}{l}\text { Lung adenocarcinoma, } \\
\text { risk, association with }\end{array}$ & $X P A$ & $\mathrm{~T}>\mathrm{C}: \mathrm{CC}$ & & Ancestral \\
\hline CR020828 & chr9 & 106730356 & - & DP & $\begin{array}{l}\text { Reduced risk of } \\
\text { coronary artery } \\
\text { disease, association } \\
\text { with }\end{array}$ & $A B C A I$ & $\mathrm{G}>\mathrm{C}: \mathrm{CC}$ & & Ancestral \\
\hline CR052068 & chr9 & $1369 \mid 1887$ & + & FP & $\begin{array}{l}\text { Promoter activity, } \\
\text { association with }\end{array}$ & FCN2 & $A>G: G G$ & & Ancestral \\
\hline CR042847 & chr9 & 138995962 & + & DP & $\begin{array}{l}\text { HDL cholesterol, } \\
\text { association with? }\end{array}$ & PTGDS & $A>C: C C$ & & Ancestral \\
\hline
\end{tabular}

